\title{
50 years of amino acid hydrophobicity scales: revisiting the capacity for peptide classification
}

\author{
Stefan Simm¹, Jens Einloft ${ }^{2}$, Oliver Mirus ${ }^{1}$ and Enrico Schleiff ${ }^{3 *}$
}

\begin{abstract}
Background: Physicochemical properties are frequently analyzed to characterize protein-sequences of known and unknown function. Especially the hydrophobicity of amino acids is often used for structural prediction or for the detection of membrane associated or embedded $\beta$-sheets and a-helices. For this purpose many scales classifying amino acids according to their physicochemical properties have been defined over the past decades. In parallel, several hydrophobicity parameters have been defined for calculation of peptide properties. We analyzed the performance of separating sequence pools using 98 hydrophobicity scales and five different hydrophobicity parameters, namely the overall hydrophobicity, the hydrophobic moment for detection of the a-helical and $\beta$-sheet membrane segments, the alternating hydrophobicity and the exact $B$-strand score.

Results: Most of the scales are capable of discriminating between transmembrane a-helices and transmembrane $\beta$-sheets, but assignment of peptides to pools of soluble peptides of different secondary structures is not achieved at the same quality. The separation capacity as measure of the discrimination between different structural elements is best by using the five different hydrophobicity parameters, but addition of the alternating hydrophobicity does not provide a large benefit. An in silico evolutionary approach shows that scales have limitation in separation capacity with a maximal threshold of 0.6 in general. We observed that scales derived from the evolutionary approach performed best in separating the different peptide pools when values for arginine and tyrosine were largely distinct from the value of glutamate. Finally, the separation of secondary structure pools via hydrophobicity can be supported by specific detectable patterns of four amino acids.

Conclusion: It could be assumed that the quality of separation capacity of a certain scale depends on the spacing of the hydrophobicity value of certain amino acids. Irrespective of the wealth of hydrophobicity scales a scale separating all different kinds of secondary structures or between soluble and transmembrane peptides does not exist reflecting that properties other than hydrophobicity affect secondary structure formation as well. Nevertheless, application of hydrophobicity scales allows distinguishing between peptides with transmembrane a-helices and $\beta$-sheets. Furthermore, the overall separation capacity score of 0.6 using different hydrophobicity parameters could be assisted by pattern search on the protein sequence level for specific peptides with a length of four amino acids.
\end{abstract}

Keywords: Hydrophobicity scale, Transmembrane sheets, Transmembrane helix, Beta-sheet, Amino acid pattern, Alternate hydrophobicity

\footnotetext{
*Correspondence: schleiff@bio.uni-frankfurt.de

${ }^{3}$ Department of Biosciences, Molecular Cell Biology of Plants, Cluster

of Excellence Frankfurt (CEF) and Buchmann Institute of Molecular Life

Sciences (BMLS), Goethe University, Max von Laue Str. 9, 60438 Frankfurt/

Main, Germany

Full list of author information is available at the end of the article
} 


\section{Background}

Hydrophobicity as a physicochemical property is frequently used to characterize secondary structures of proteins. Early on it was noted that this property of amino acids dominates the initial interactions during protein folding $[1,2]$. In addition, the physicochemical properties of secondary structures depend on the properties of their amino acids and differ in relation to the native environment of the secondary structure, e.g., in solution or in membranes [3-5]. Considering this, it is not of surprise that the classification and characterization of amino acids according to their hydrophobicity attracted much attention.

In 1962 the first hydrophobicity scale of amino acids was formulated [6]. In addition, a first model to calculate the difference in free energy for the unfolded and native form of the protein catalase in solution was established [6]. Ever since many "hydrophobicity scales" were published. However, not all of these scales focus exclusively on hydrophobicity, but we will continue using this term. The information about hydrophobicity for the amino acids were extracted from biochemical experiments [7], distributions of amino acids in different protein classes $[8,9]$, the capacity of amino acids to participate in hydrophobic or hydrophilic milieu $[10,11]$ or from in silico calculations [12]. Today, about 98 "hydrophobicity scales" exist which contain a defined hydrophobicity value for each of the 20 amino acids. A high variance between these scales can be expected due to the variance of the underlying experimental approaches.

At the same time many hydrophobicity parameters for peptide classification have been developed for specific purposes. The overall hydrophobicity was introduced to globally classify peptides [6]. In addition, a hydrophobic moment for detection of the helical membrane segments [13], the alternating hydrophobicity for detection of membrane embedded $\beta$-sheets $[14,15]$ or exact $\beta$-strand score (EBSS) considering the frequency of amino acids pointing inward or outward of a $ß$-barrel [16] has been defined.

In parallel many alternative algorithms and methods have been developed to predict protein properties based on hydrophobicity scales and classify them concerning environment (soluble, transmembrane) or function. Among them are routines for the prediction of transmembrane regions [17-20] or protein folding [21-25]. Even today, the hydrophobicity scales are often used to define properties of peptides within proteins [26-29]. However, the wealth of hydrophobicity scales complicates the process of scale selection and of the parameters to be calculated.

Thus, 50 years after formulation of the first scale we analyzed 98 different hydrophobicity scales present in the literature $[22,30,31]$. We used the overall hydrophobicity, the hydrophobic moment for detection of $\alpha$-helical and $\beta$-sheet transmembrane elements, the alternating hydrophobicity and the EBSS as parameters to evaluate their influences on the separation on different secondary structure pools. For the analysis of the different scales and parameters we developed a five dimensional consensus approach to define the quality of the combinatory usage. Finally, we clustered the hydrophobicity scales to classify their performance for general separation capacity of secondary structures, environmental specifications or subsets thereof. We found that the overall performance of the hydrophobicity scales is rather comparable irrespective of the strategy of generation. However, the application of more than one hydrophobicity parameter enhances the capacity of the pool separation, but the alternating hydrophobicity has the lowest impact on the separation capacity when compared with the other four parameters. In general hydrophobicity is suitable to classify transmembrane $\alpha$-helices and $\beta$-sheets better than peptides with other secondary structures. However, specific pattern of four or five amino acids were identified in the different peptide pools analyzed.

\section{Results and discussion}

\section{Sequence pools, hydrophobicity scales and parameter selection}

Different sequence pools were generated to study the separation capacity of hydrophobicity scales and hydrophobicity parameters. To this end, sequences of proteins with known structure were extracted from the ASTRAL40 (http://scop.berkeley.edu/astral/) database [32] and dissected in sequences with exclusive $\alpha$-helical, $ß$-sheet and random coil (random) content. The $\alpha$-helical and $ß$-sheet sequences were further separated in pools representing transmembrane segments (tm-sheet, tm-helix) and soluble (s-sheet. s-helix; annotated as cytoplasmic). Subsequently, the two small individual transmembrane pools were expanded by one round of psi-blast using the sequences with structural information as bait. Psi-blast for the two small datasets using only sequences of known secondary structures was performed to reach an increase of highly similar sequences from the bait sequence pools. To prevent overfitting of the two pools, filtering for redundant and similar (>95\% sequence identity) sequences was performed. This approach was required to avoid artifacts by comparing drastically different volumes and peptide densities. Otherwise, a small volume could result in an unjustified good separation value. The other three pools (random, s-helix, s-sheet) were not expanded leading to the final sequence number for the five different secondary structure pools (tm-sheet, tm-helix, s-sheet, s-helix, random) (Table 1; Additional file 1: Table S1, Additional file 2: Table S2). 
Table 1 Sequence pools based on secondary structure dissection

\begin{tabular}{|c|c|c|c|}
\hline Abbr. & Sequences & Description & Name \\
\hline random & 16447 & $\begin{array}{l}\text { Non-transmembrane } \\
\text { random coils }\end{array}$ & Random coil \\
\hline s-sheet & 8134 & $\begin{array}{l}\text { Non-transmembrane } \\
\beta \text {-sheets }\end{array}$ & Soluble $\beta$-sheets \\
\hline s-helix & 34452 & $\begin{array}{l}\text { Non-transmembrane } \\
\text { a-helices }\end{array}$ & Soluble a-helices \\
\hline tm-sheet & 4407 & $\begin{array}{l}\text { Transmembrane } \\
\beta \text {-sheets }\end{array}$ & $\begin{array}{l}\text { Trans-membrane } \\
\beta \text {-sheets }\end{array}$ \\
\hline tm-helix & 9922 & $\begin{array}{l}\text { Transmembrane } \\
\text { a-helices }\end{array}$ & $\begin{array}{l}\text { Trans-membrane } \\
\text { a-helices }\end{array}$ \\
\hline
\end{tabular}

All peptides have a minimal length of ten amino acids required for EBBS based analysis. Soluble pools contain only peptides with the defined SSE, while the trans-membrane pools contain both, peptides with the given SSE only and with the given SSE and additional amino acids to reach a length of ten amino acids

Further, we implemented an in silico tryptic [K (Lysine)/R (Arginine)] digest of the whole ASTRAL40 [32] database. On the one hand, this approach yields peptides with mixed structural content. On the other hand, on the base of these peptides we wanted to test whether peptides identified by mass spectrometric approaches typically generated by tryptic digest can be used to define topologies of proteins. These sequences were classified according to their continuous (dc-sheet, dc-helix, dcrandom) or discontinuous (dd-sheet, dd-helix, dd-random) dominating secondary structure elements (SSE). Peptides without dominating SSE are clustered concerning their portion of different SSE (no-helix, no-sheet, no-random, all). In addition, sequences with transmembrane content were pooled individually for the SSE helix and sheet (krtm-helix, krtm-sheet, Table 2). Combining the pools generated by in silico tryptic digestion or based on known secondary structure approaches, we ended up with 17 different sequence pools. Each of the 17 different sequence pools contains a discrete number of sequences (Tables 1, 2).

After gathering the test pools different hydrophobicity scales were extracted from literature (Tables 3, 4; Additional file 1: Table S1). The 98 selected scales cover experimentally developed scales, calculated scales as well as scales which have been created by improving pre-existing scales. In addition, eight scales represent the reverse algebraic numeral scales to other scales to test if the algebraic numeral has an influence on the results.

To investigate the separation capacity of the selected hydrophobicity scales on the different defined sequence pools five measurement parameters have been defined (Table 5). The EBSS [16], the alternating hydrophobicity $[14,15]$ and the hydrophobic moment calculated for $\beta$-sheets with an angle of $180^{\circ}$ [13] are hallmarks for hydrophobic content, while the hydrophobic moment calculated for $\alpha$-helices [14] and the average hydrophobicity [33] are used to identify $\alpha$-helical transmembrane regions (hydrophobic-moment $\alpha$ ). Each of the parameters was calculated in a sliding window of ten amino acids. The largest and smallest value for each peptide was considered as outlined below.

\section{The relation of the hydrophobicity scales}

We clustered the hydrophobicity scales by comparing the hydrophobicity value for each amino acid to each other. The variance of the different hydrophobicity scales was analyzed by Pearson correlation. The

Table 2 Sequence pools based in silico K/R-digestion

\begin{tabular}{|c|c|c|c|}
\hline Abbr. & Sequences & & Description \\
\hline dc-helix & 8208 & Dominating SSE & a-helix; continuous \\
\hline dc-sheet & 1978 & & $\beta$-sheet; continuous \\
\hline dc-random & 4490 & & random coil; continuous \\
\hline dd-helix & 5154 & & a-helix; discontinuous \\
\hline dd-sheet & 3322 & & $\beta$-sheet; discontinuous \\
\hline dd-random & 2331 & & random coil; discontinuous \\
\hline no-helix & 11039 & No dominating SSE & $\beta$-sheets and random coils \\
\hline no-sheet & 7770 & & a-helices and random coils \\
\hline no-random & 281 & & $\beta$-sheets and $a$-helices \\
\hline all & 22237 & & $\beta$-sheets, a-helices, and random coils \\
\hline krtm-helix & 214 & & TM a-helices with additional AA at $\mathrm{N}$ - or C-terminus \\
\hline krtm-sheet & 119 & & TM $\beta$-sheets with additional AA at $\mathrm{N}$ - or $\mathrm{C}$-terminus \\
\hline
\end{tabular}

All peptides have a minimal length of ten amino acids (AA). Dominating SSE stands for SSE content larger than $70 \%$ of all AA. Continuous means that this SSE is without gap

$T M$ transmembrane. The nomenclature is as follows: $d c$ peptide derived by in silico tryptic digest with continuous structural element, $d d$ peptide derived by in silico tryptic digest with discontinuous structural element, $\mathrm{krtm}$ peptide derived by in silico tryptic digest (kr) with transmembrane segment 
Table 3 Improved, calculated and inverted hydrophobicity scales

\begin{tabular}{|c|c|c|c|c|c|}
\hline & ID & Year & Author & Scale & Ref. \\
\hline \multirow[t]{22}{*}{ Improved } & 65 & 1968 & Zimmerman & ZIMJ680101 & [36] \\
\hline & 54 & 1971 & Tanford and Nozaki & NOZY710101 & [37] \\
\hline & 29 & 1975 & Jones & JONES & [35] \\
\hline & 63 & 1975 & Jones & JOND750101 & [35] \\
\hline & 78 & 1976 & Levitt & LEVIT & [44] \\
\hline & 27 & 1982 & Kyte and Doolittle & KYTJ820101 & [38] \\
\hline & 12 & 1983 & $\begin{array}{l}\text { Sweet and } \\
\text { Eisenberg }\end{array}$ & SWER830101 & [42] \\
\hline & 83 & 1983 & $\begin{array}{l}\text { Sweet and } \\
\text { Eisenberg }\end{array}$ & SWEET & [42] \\
\hline & 3 & 1984 & Eisenberg & ESID840101 & [45] \\
\hline & 51 & 1984 & Eisenberg & EISEN & [45] \\
\hline & 47 & 1985 & Guy & GUYH850101 & [46] \\
\hline & 74 & 1985 & Guy & GUYFE & [46] \\
\hline & 80 & 1985 & Rose & ROSEF & [47] \\
\hline & 57 & 1985 & Rose & ROSG850102 & [47] \\
\hline & 82 & 1987 & Cornette & NNEIG & [41] \\
\hline & 41 & 1989 & Cohen and Kuntz & COHEN & [48] \\
\hline & 44 & 1998 & Juretic & MDKO & [22] \\
\hline & 45 & 1998 & Juretic & MDK1 & [22] \\
\hline & 24 & 1998 & Juretic & JURD980101 & [22] \\
\hline & 89 & 2005 & Zviling & SET1 & [12] \\
\hline & 90 & 2005 & Zviling & SET2 & [12] \\
\hline & 91 & 2005 & Zviling & SET3 & [12] \\
\hline \multirow[t]{11}{*}{ Calculated } & 38 & 1976 & Chothia & CHOTA & [49] \\
\hline & 50 & 1976 & Chothia & CHOC760103 & [49] \\
\hline & 75 & 1976 & Chothia & $\mathrm{CHOTH}$ & [49] \\
\hline & 72 & 1980 & Ponnuswamy & PONNU & {$[8]$} \\
\hline & 81 & 1983 & $\begin{array}{l}\text { Sweet and } \\
\text { Eisenberg }\end{array}$ & $\begin{array}{l}\text { Sweet and Eisen- } \\
\text { berg }\end{array}$ & [41] \\
\hline & 36 & 1985 & Kidera & KIDER & [50] \\
\hline & 39 & 1985 & Rose & ROSEB & [47] \\
\hline & 55 & 1985 & Welling & Welling & [51] \\
\hline & 53 & 1986 & Rao and Argos & Rao and Argos & [52] \\
\hline & 70 & 1989 & Fasman & FASG890101 & [48] \\
\hline & 79 & 1989 & Fasman & GIBRA & [48] \\
\hline \multirow[t]{11}{*}{ Inverted } & 92 & 1973 & Bull and Breese & BULDG reverse & [53] \\
\hline & 64 & 1976 & Levitt & LEVM760101 reverse & [54] \\
\hline & 95 & 2001 & Bishop & Bishop reverse & [55] \\
\hline & 59 & 1996 & Wimley and White & Wimley reverse & [56] \\
\hline & 94 & 1985 & Welling & Welling reverse & [51] \\
\hline & 26 & 1986 & Engelman & $\begin{array}{l}\text { ENGD860101 } \\
\text { reverse }\end{array}$ & [39] \\
\hline & 40 & 1985 & Rose & ROSEA reverse & [47] \\
\hline & 21 & 1995 & Wilce & WILM950103 reverse & {$[7]$} \\
\hline & 23 & 1995 & Kuhn & KUHL950101 reverse & [57] \\
\hline & 11 & 1990 & Prabhakaran & $\begin{array}{l}\text { PRAM900101 } \\
\text { reverse }\end{array}$ & [58] \\
\hline & 96 & 1989 & Fasman & FASG890101 reverse & [48] \\
\hline
\end{tabular}

Table 3 continued

\begin{tabular}{|c|c|c|c|c|}
\hline ID & Year & Author & Scale & Ref. \\
\hline 93 & 1985 & Guy & $\begin{array}{l}\text { GUYH850101 } \\
\text { reverse }\end{array}$ & [46] \\
\hline 25 & 1985 & Kidera & KIDA850101 reverse & {$[50]$} \\
\hline 98 & 1988 & Roseman & $\begin{array}{l}\text { ROSM880102 } \\
\text { reverse }\end{array}$ & [59] \\
\hline 88 & 1988 & Roseman & $\begin{array}{l}\text { ROSM880103 } \\
\text { reverse }\end{array}$ & [59] \\
\hline 97 & 1988 & Roseman & $\begin{array}{l}\text { ROSM880101 } \\
\text { reverse }\end{array}$ & [59] \\
\hline 84 & 1981 & Wolfenden & $\begin{array}{l}\text { WOLR810101 } \\
\text { reverse }\end{array}$ & {$[60]$} \\
\hline
\end{tabular}

Shown are the category of the scale (column 1), the ID of the hydrophobicity scale (column 2) the year of the publishing (column 3), the name of the authors (column 4) and the name of the scale (column 5)

values obtained were used to calculate the dissimilarity $\left(=\sqrt{1-\text { correlation }^{2}}\right)$ to create an Unweighted Pair Group Method with Arithmetic mean (UPGMA) tree of the hydrophobicity scales via MEGA6 [34]. The tree was used to cluster those scales to groups of similar amino acid value behavior setting a threshold (Fig. 1; Additional file 2: Table S2). The linearized UPGMA tree of the 98 hydrophobicity scales was inspected to split the scales in clusters using a threshold of a maximal dissimilarity of 0.05 . The created clusters were named alphabetically. The UPGMA tree was circled to give an overview of all 98 hydrophobicity scales and their position at a glance (Fig. 1).

As expected, the hydrophobicity scales generated by inverting the amino acid values cluster with the original scales (Table 3). However, not all hydrophobicity scales that have been created by the same experimental approach or by the same author cluster together (Table 4). Most prominent examples are (i) the scales generated by Jones (scales 29, 63; JONES, JOND750101; cluster K) [35] which adjusted the scale of Zimmerman (scale 69; ZIMJ680101; cluster J) [36] by considering experimental derived values (scale 54, Tanford; cluster A) [37]; or (ii) the scales proposed by Zviling (scales 89-91; SET1-3; cluster I) [12] that have been based on the scales of Kyte and Doolittle (cluster B) [38] and Engelman (cluster A) [39].

Calculation of separation capacity of hydrophobicity scales Next we analyzed the capacity of the 98 hydrophobicity scales to separate the 17 defined sequence pools. The initial analysis was based on all five hydrophobicity parameters. For each parameter the maximal and minimal value for each peptide was calculated (Table 5). However, we realized that the simultaneous application of the minimal and maximal value of the same parameter 
Table 4 Experimental hydrophobicity scales

\begin{tabular}{|c|c|c|c|c|}
\hline ID & Year & Author & Scale & Ref. \\
\hline 46 & 1973 & Bull and Breese & BULDG & {$[53$} \\
\hline 4 & 1978 & Manavalan and Ponnuswamy & MANP780101 & [61] \\
\hline 76 & 1979 & Heijne and Bloomberg & VHEG790101 & {$[6$} \\
\hline 52 & 1979 & Janin & JANJ790102 & {$[6$} \\
\hline 73 & 1979 & Janin & JANIN & \\
\hline 37 & 1979 & Wolfenden and Cullis & WOLR790101 & \\
\hline 5 & 1980 & Ponnuswamy & PONP800101 & [8] \\
\hline 6 & 1980 & Ponnuswamy & PONP800102 & [8] \\
\hline 7 & 1980 & Ponnuswamy & PONP800103 & [8] \\
\hline 8 & 1980 & Ponnuswamy & PONP800104 & [8] \\
\hline 9 & 1980 & Ponnuswamy & PONP800105 & [8] \\
\hline 10 & 1980 & Ponnuswamy & PONP800106 & [8] \\
\hline 49 & 1981 & Wilson & Wilson & \\
\hline 61 & 1982 & Argos & ARGP820101 & \\
\hline 62 & 1983 & Fauchere and Pliska & FAUJ830101 & {$[1$} \\
\hline 71 & 1983 & Fauchere and Pliska & $\mathrm{FAUCH}$ & \\
\hline 48 & 1985 & Miyazawa and Jerningen & MIYS850101 & {$[6$} \\
\hline 28 & 1986 & Engelman & ENGEL & \\
\hline 77 & 1988 & Roseman & ROSEM & {$[3$} \\
\hline 42 & 1989 & Jacobs and White & JACWH & {$[67$} \\
\hline 56 & 1990 & Parker & PARJ860101 & {$[68$} \\
\hline 85 & 1990 & Cowan and Whittacker & Cowan Whittacker & \\
\hline 86 & 1988 & Roseman & ROSM880101 & \\
\hline 87 & 1988 & Roseman & ROSM880102 & {$[5$} \\
\hline 68 & 1990 & Cowan and Whittacker & COWR900101 & {$[6$} \\
\hline 69 & 1991 & Black and Mould & BLAS910101 & {$[7$} \\
\hline 43 & 1992 & Cassari and Sippl & CASSI & \\
\hline 1 & 1992 & Cid & CIDH920101 & [9] \\
\hline 2 & 1992 & Cid & $\mathrm{CIDH} 920105$ & [9] \\
\hline 30 & 1992 & Cid & CIDBB & [9] \\
\hline 31 & 1992 & Cid & $\mathrm{CIDA}+$ & [9] \\
\hline 32 & 1992 & Cid & CIDAB & 50 \\
\hline 33 & 1993 & Ponnuswamy and Gromiha & PONG1 & {$[72$} \\
\hline 34 & 1993 & Ponnuswamy and Gromiha & PONG2 & ] \\
\hline 35 & 1993 & Ponnuswamy and Gromiha & PONG3 & \\
\hline 67 & 1993 & Ponnuswamy and Gromiha & PONP930101 & 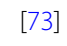 \\
\hline 19 & 1995 & Wilce & WILM950101 & {$[7]$} \\
\hline 20 & 1995 & Wilce & WILM950102 & {$[7]$} \\
\hline 22 & 1995 & Wilce & WILM950104 & [7 \\
\hline 60 & 1996 & Wimley and White & Wimley & \\
\hline 58 & 2001 & Bishop & Bishop & {$[5$} \\
\hline 13 & 2001 & Naderi-Manesh & NADH010101 & {$[4$} \\
\hline 14 & 2001 & Naderi-Manesh & NADH010102 & \\
\hline 15 & 2001 & Naderi-Manesh & NADH010103 & \\
\hline 16 & 2001 & Naderi-Manesh & NADH010104 & \\
\hline 17 & 2001 & Naderi-Manesh & NADH010106 & {$[40$} \\
\hline 18 & 2001 & Naderi-Manesh & NADH010107 & $\Gamma$ \\
\hline 66 & 2001 & Naderi-Manesh & NADH010105 & 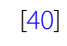 \\
\hline
\end{tabular}

Shown are the ID of the hydrophobicity scale (column 1), the year of the publishing (column 2), the name of the authors (column 3 ) the name of the scale (column 4) and a relevant reference the scale was extracted from does not increase the separation performance. By this we limit the parameter selection too either the minimal or the maximum value. We calculated the 32 parameter combinations (five parameter and alternating minimal and maximal value) for each peptide for all 98 hydrophobicity scales. The resulting five dimensional vectors for each peptide and each hydrophobicity scale were used to define five dimensional clouds for each pool and each specific hydrophobicity scale.

For the analysis of the separation capacity (Fig. 2, right) of a scale between two clouds of sequence pools we calculated the overlap volume (Fig. 2, left) and the number of peptides within the overlap (Fig. 2, middle). The size of the overlap volume and the number of peptides within the overlap volume negatively correlates with the separation capacity for two sequence pools. Further, we defined the "convex envelope" as described in the "Methods" section. Next we removed all peptides, which are part of the convex envelope. We recalculated the volumes of the pools and repeated the last step of the routine with the new volumes. Removing the peptides on the convex envelope was performed because the presence of only few peptides positioned distantly from the other peptides could increase the volume drastically. In case that the peptides are positioned close to others the volume did not change significantly and thus, this step improved the reliability of assignment of the majority of peptides.

Next, we defined a separation capacity score (Formula 1) to rank all scenarios. $S_{v}$ is a score based on the volume of the overlap in relation to the volumes of the two clouds. For $S_{p}$ we counted all peptides in the overlap volume of two sequence pools and set them in relation to all peptides of the two clouds. The score $\mathrm{S}$ is scaled between zero (both clouds totally overlap) and one (no peptides in overlap volume) and gives the quality of a certain hydrophobicity scale for the separation of two defined pools.

Formula 1 Separation capacity score

$$
\begin{aligned}
& S=S_{v} * S_{p} ; \quad S_{v}=1-\left(2 * \frac{V_{o v}}{\left(V_{1}+V_{2}\right)}\right) ; \\
& S_{p}=1-\left(\frac{P_{o v}}{\left(P_{1}+P_{2}\right)}\right)
\end{aligned}
$$

Here, $\mathrm{P}_{1}$ and $\mathrm{P}_{2}$ are the total numbers of peptides of pool 1 and $2, \mathrm{P}_{o v}$ is the number of all sequences in the overlap volume, $V_{1}$ and $V_{2}$ are the volumes defined by the sequence pools 1 and 2 , and $\mathrm{V}_{o v}$ is the overlapping volume of both pools. The number of $\mathrm{V}_{\mathrm{i}}$ and $\mathrm{P}_{\mathrm{i}}$ was always $\mathrm{i}=2$ because two pools were analyzed in parallel.

The general $S$ value was calculated for each scale for the sequence pools based on secondary structure 
Table 5 Hydrophobicity parameters

\begin{tabular}{lll}
\hline Index & Name & Description \\
\hline 0 & Max. exact $\beta$-strand score (EBSS) & Parameter to score the probability of a sequence with $\geq 10$ AA to be a TM $\beta$-sheet [16] \\
1 & Min. exact $\beta$-strand score (EBSS) & \\
2 & Max. alternating hydrophobicity & High alternating hydrophobicity probing for polar and unpolar AA alternation typical for trans- \\
3 & Min. alternating hydrophobicity & membrane $\beta$-sheets [14, 15] \\
4 & Max. hydrophobicity-moment a & Analyzing the distribution of hydrophobicity considering the amino acid distribution in a-helices \\
5 & Min. hydrophobicity-moment a & with an angle between amino acids of $100^{\circ}$ to probe for potential to form a TM a-helix [13] \\
6 & Max. hydrophobicity-moment $\beta$ & Analyzing the distribution of hydrophobicity considering the amino acid distribution of $\beta$-sheets \\
7 & Min. hydrophobicity-Moment $\beta$ & with an angle between amino acids of 180 to probe for potential to form a TM $\beta$-sheet [13] \\
8 & Max. average hydrophobicity & Average hydrophobicity of the peptide [33] \\
9 & Min. average hydrophobicity & \\
\hline
\end{tabular}

Shown are the index (column 1), the name (column 2) and the description of the used hydrophobicity parameters (column 3 ). The description is equal for the minimum and maximum of the used hydrophobicity parameter

dissection (Table 1; Fig. 3a, orange line), the sequence pools generated by in silico digestion (Table 2; Fig. 3a, blue line) and both together, the sequence pools based on secondary structure dissection and the sequence pools generated by in silico digestion (Fig. 3a, green line: mixed). While all scales perform similar for the pool derived by digestion or by combining all pools, a distinction is found for the "structure" pool. The three best performing hydrophobicity scales are 14, 15 (NADH010102, NADH010103; Table 4) [40] and 82 (NNEIG; Table 3) [41]. Scales 14 and 15 are experimental hydropathic scales and based on self-information values of a two state model with 9 and $16 \%$ protein surface accessibility. Scale 82 is an improvement of the scale of Sweet and Eisenberg from 1983 [42] using the eigenvalues of a normalized nearest neighbor matrix.

In parallel, the average $S$ value for the clusters of hydrophobicity scales (Fig. 1) defined according to the UPGMA-tree was calculated (Fig. 3b). The average S values observed for the peptide pools obtained by in silico tryptic digest (Fig. 3b, blue) or by the combination of peptide pools generated by the two strategies (Fig. 3b green) do not show a dependence on the selected cluster. Only for the secondary structure peptide pools the observed average $\mathrm{S}$ values differ between 0.28 for the cluster B and 0.13 for cluster X. Moreover, after sorting the clusters according to the average $S$ values for the secondary structure peptide pools the order of clusters does not follow the order in the UPGMA tree (Fig. 3b, orange).

\section{Separation of specific structure pools via hydrophobicity}

The moderate separation capacity of all hydrophobicity scales using all 17 sequence pools prompted us to inspect the separation of the individual sequence pool pairs. The results are exemplified for the separation value for the best performing scale 14 [40] (Fig. 4a) as well as the maximal separation capacity out of all 98 hydrophobicity scales for each pairwise sequence pool combination (Fig. 4b). Globally, the $S$ values obtained for the pairwise pool separation by maximal separation capacity out of all 98 hydrophobicity scales (Fig. 4b) are in general larger than the $S$ values obtained by using the best performing scale 14 only.

In detail, the three pools with transmembrane $\alpha$-helix (krtm-helix), with transmembrane $\beta$-sheet (krtm-sheet) or without random coil content (no-random) generated by digestion have the largest $S$ value while analyzing the overlap with other sequence pools, irrespective whether the best scale (Fig. 4a) or the best value (Fig. 4b) is considered. In contrast, the secondary structure transmembrane pools (tm-sheet, tm-helix) show low $\mathrm{S}$ values while analyzing the overlap with other pools. Nevertheless, the $S$ values of the secondary structure transmembrane pools are larger than the $S$ values found while analyzing the overlap of the remaining sequence pools (Fig. 4b).

Remarkably, high $\mathrm{S}$ values were found when the overlap between the two secondary structural transmembrane pools (tm-sheet, tm-helix) and the three pools with transmembrane $\alpha$-helix (krtm-helix), with transmembrane $\beta$-sheet (krtm-sheet) or without random coil content (no-random) generated by digestion was calculated. This might suggest that the regions flanking the transmembrane domain present in the sequences of the peptide pools generated by digestion provide additional information. This information in combination with the hydrophobicity might give an additional signature for such domains. Hence, in silico digestion with subsequent analysis by the described parameters using e.g. the hydrophobicity scale 14 can be used to detect transmembrane helices and sheets.

With respect to the remaining pools we observed that the $S$ value obtained while analyzing the overlap of 


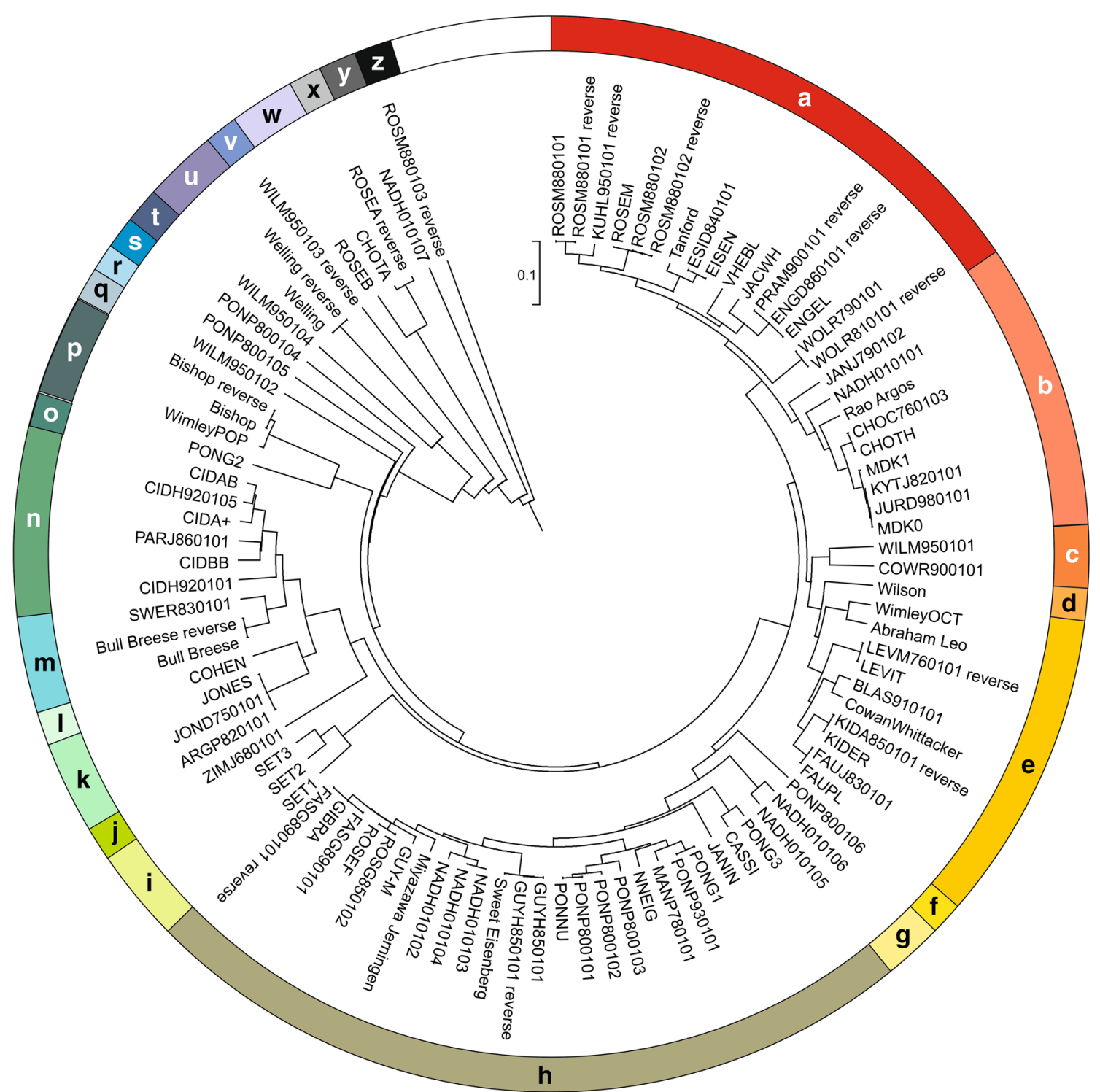

Fig. 1 Clustering of hydrophobicity scales. Shown is the UPGMA tree of the clustered hydrophobicity scales based on the normalized amino acid value distances (see "Methods" section). The hydrophobicity scales are clustered to groups ( $a$ to $z$ ) within similarity larger than 0.07

the secondary structure pools (s-sheet, s-helix and random) is higher when compared to the pools containing sequences with mixed structures (Fig. 4a, b). This result is expected, as the chosen parameters detect the individual elements and a mixture thereof yields mixed information.

Consequently, we analyzed the performance of the individual hydrophobicity parameters and the usage of the multi-dimensional approach. We realized that the $\mathrm{S}$ value is more dependent on the combination of the hydrophobicity parameter in a multi-dimensional vector than on a specific hydrophobicity scale (Additional file 3: Fig. S1).
Including the top $5 \%$ of all scenarios for separating two pools from each other (Fig. 5a) the influence of the different parameter varies significantly (Fig. 5b). It becomes obvious that the average hydrophobicity and EBSS have the strongest impact on the separation quality, while the alternating hydrophobicity previously thought to specifically recognize transmembrane $\beta$-strands $[14,15]$ has the lowest impact on separation. Only for the mixed situation we observed that the alternating hydrophobicity has no impact at all. Thus, the overall performance is dependent on all parameter although to a different extent. 

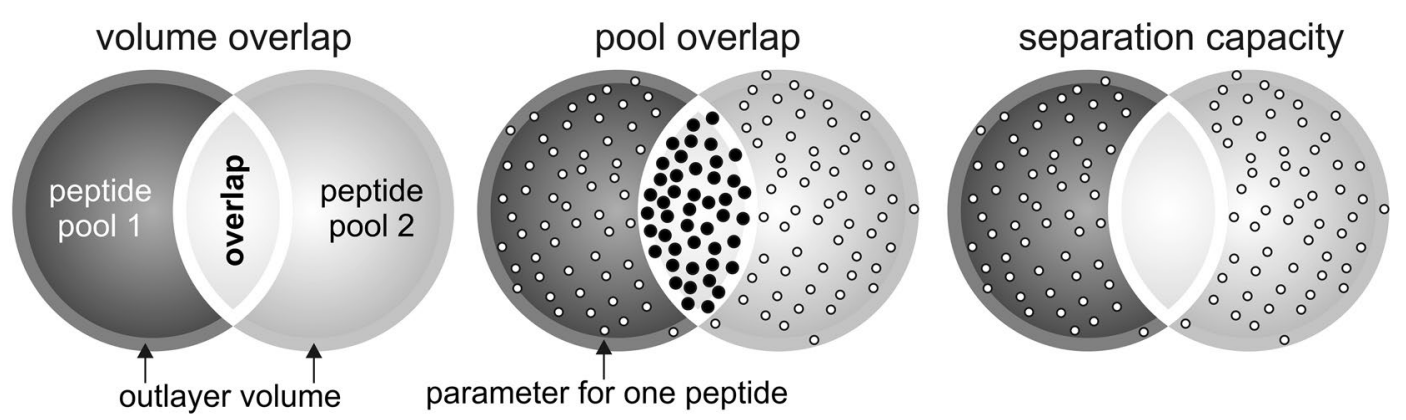

Fig. 2 Scoring scheme. Shown is the scoring scheme of the separation of two sequence pools. Both sequence pools (dark grey area, grey area) build a cloud. The overlap of both clouds (light grey area) as well as the sequences (bold points) in this overlap is used to calculate the separation capacity. The circle surrounding both clouds represents the volume of the outlayer

\section{Benefit of amino acid pattern to separate specific structure pools}

An amino acid based approach for the different structure pools was subsequently considered in addition to the hydrophobicity based separation. At first the amino acid composition of the different pools was analyzed, which did not yield a significant difference between the individual pools (Additional file 4: Table S3).

Thus, the occurrence of amino acid patterns of two to five amino acids was analyzed utilizing a markov chain approach (Fig. 6; "Methods" section). Nearly all ( 80 \%) detected amino acid patterns up to the length of three occur in each of the different sequence pools. The number of globally occurring amino acid patterns of a length of four drops down to $60 \%$. An elongation of the pattern length to more than five amino acids results in coverage of less than $5 \%$ and thus, is not of use for separation. Thus, the exclusive appearance or at least overrepresentation of amino acid patterns of four and five amino acids in the distinct sequence pools was analyzed.

We identified several peptides of four (Table 6; Additional file 5: Table S4) and five amino acids that are specific or at least enriched in peptides of a certain pool (Table 7; Additional file 6: Table S5). Analyzing the frequency of occurrence of these peptides revealed that patterns with four amino acids are only very moderately specific as only very few are at least 50 -fold more frequent in a certain pool than in general (Table 6). In turn, patterns with five amino acids can serve as an additional discriminator because patterns with 500 -fold higher frequency in a specific pool than in the overall sequence pool exist (Table 7). This holds true particularly when the sequence pools generated by the same strategy (Tables 1 , 2) are compared. This information can only be taken in addition to the hydrophobicity parameters, because occurrence of specific amino acid patterns in one specific structure pool compared to all pools as reference did not yield an adjusted p value below 0.05 for any amino acid pattern of length five. Nevertheless, detection of $ß$-strands in peptides can be supported by the detection of penta-peptides YLVNM (dc-sheet), LTVTG, TLDGG (dd-sheet), CGGSL and YGGVT (s-sheet). Remarkably, the penta-peptides observed for the structurally derived pool is not overlapping with the penta-peptides observed for the pool derived by in silico tryptic digestion which might suggest that the latter contain specific regions at the end of the strand. Moreover, amino acid patterns specific for the transmembrane $ß$-strands are SIGA (krtmsheet, Table 6), LYGKV, PTLDL and SASAG (tm-sheet, Table 7). For peptides with mainly random content we found that S-GSSG-S, SGPSS or TILPL are enriched (random, dd-random; Table 7). In turn, for pools mainly consisting of helical structures we found only one pentapeptide specific pattern for the structural pools with $\alpha$-helix (s-helix; EELKK) and for the pool of peptides with the transmembrane $\alpha$-helix (krtm-helix; YVFFG; Table 7). Thus, a prescreening of sequence pools with these amino acid patterns might improve the classification quality.

\section{Factors influencing pool separation}

The variation of separation capacity of hydrophobicity scales (Fig. 3) prompted the analysis of the impact of individual amino acid values. However, we did not realize any correlation between specific distribution of values for individual amino acid within the individual scales and the scale performance based on the 98 known hydrophobicity scales. As an alternative approach we created random hydrophobicity scales based on the 98 already known ones (Tables 3,4). At first, the maximum and minimum amino acid values of the 98 real scales were used as interval to create 200 random hydrophobicity scales by assignment of a random value to each individual amino acid. Subsequently, several rounds of in silico evolution were 


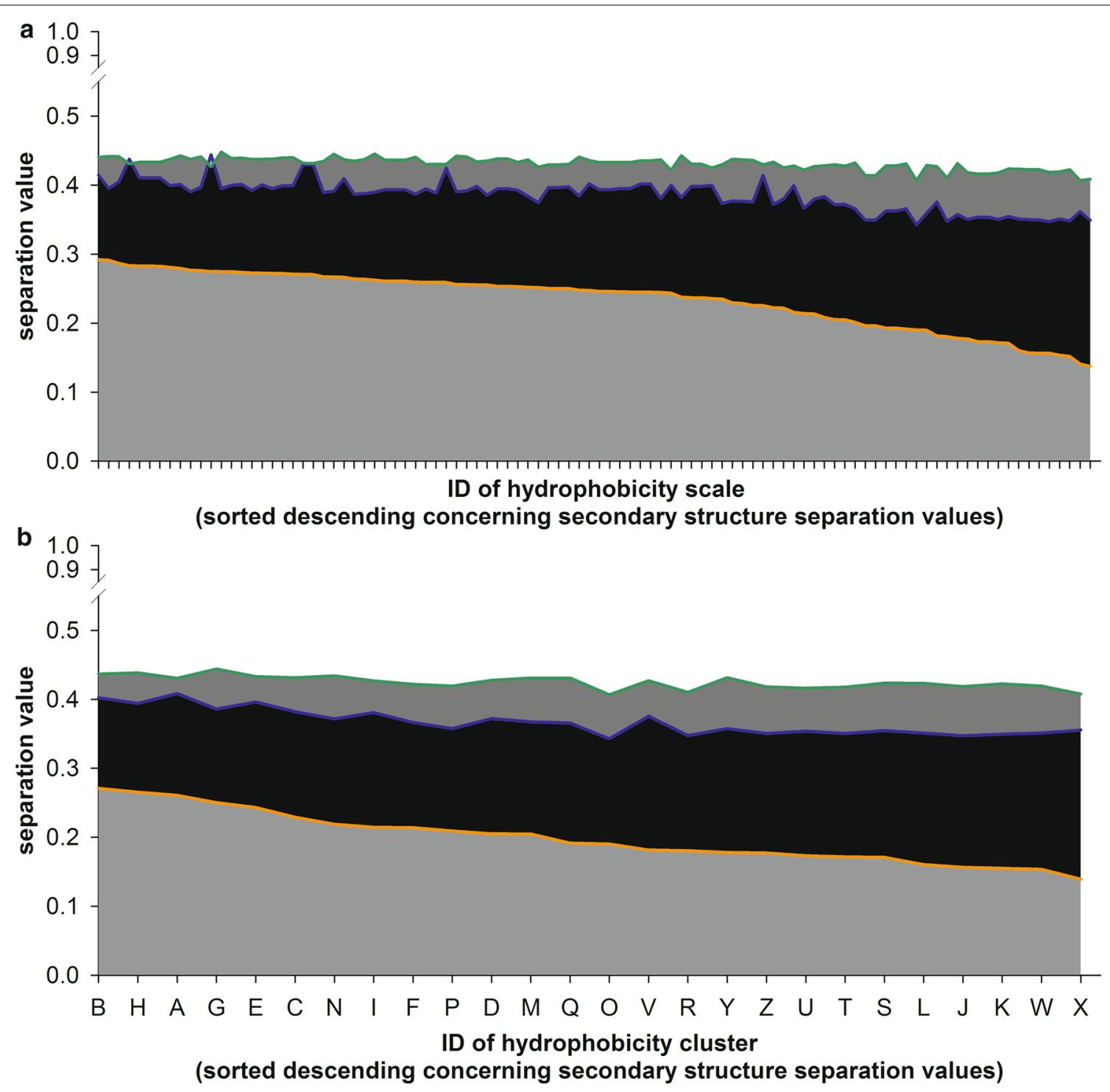

Fig. 3 Separation of pools by hydrophobicity scales. a Shown is the overall separation value for each hydrophobicity scale for the secondary structure (orange), in silico tryptic digest (blue) and mixed (green) sequence pools as area plot. The hydrophobicity scales are sorted from highest to lowest value. $\mathbf{b}$ The same as in $\mathbf{a}$ but the separation value is calculated for the cluster of hydrophobicity scales

performed to improve the separation capacity for the five different structural sequence pools (Fig. 7).

After six rounds of in silico evolution the created random hydrophobicity scales reached a separation threshold of 0.6, which is comparable to the separation potential of the best performing hydrophobicity scale. This suggests that a limit of the potential of amino acid scales for the separation of structural sequence pools exists by 0.6. Furthermore, we realized during the evolution of the hydrophobicity scales that the value of some amino acids had greater positive or negative influence on the separation capacity like others.
After establishing the evolutionary scale, we aimed at an understanding which property of a scale has an impact on its separation capacity. At first, we tested whether the general order of amino acids with respect to their hydrophobicity value is important. We realized that it is not the overall order of the amino acid hydrophobicity values that influences the performance of the hydrophobicity scale (Additional file 7: Fig. S2). At second we analyzed whether the value of specific amino acids dominate the separation capacity of a scale. We realized high $\mathrm{S}$ values for hydrophobicity scales sharing rather comparable hydrophobicity values for Gln, His, Gly, Ser or Arg to 


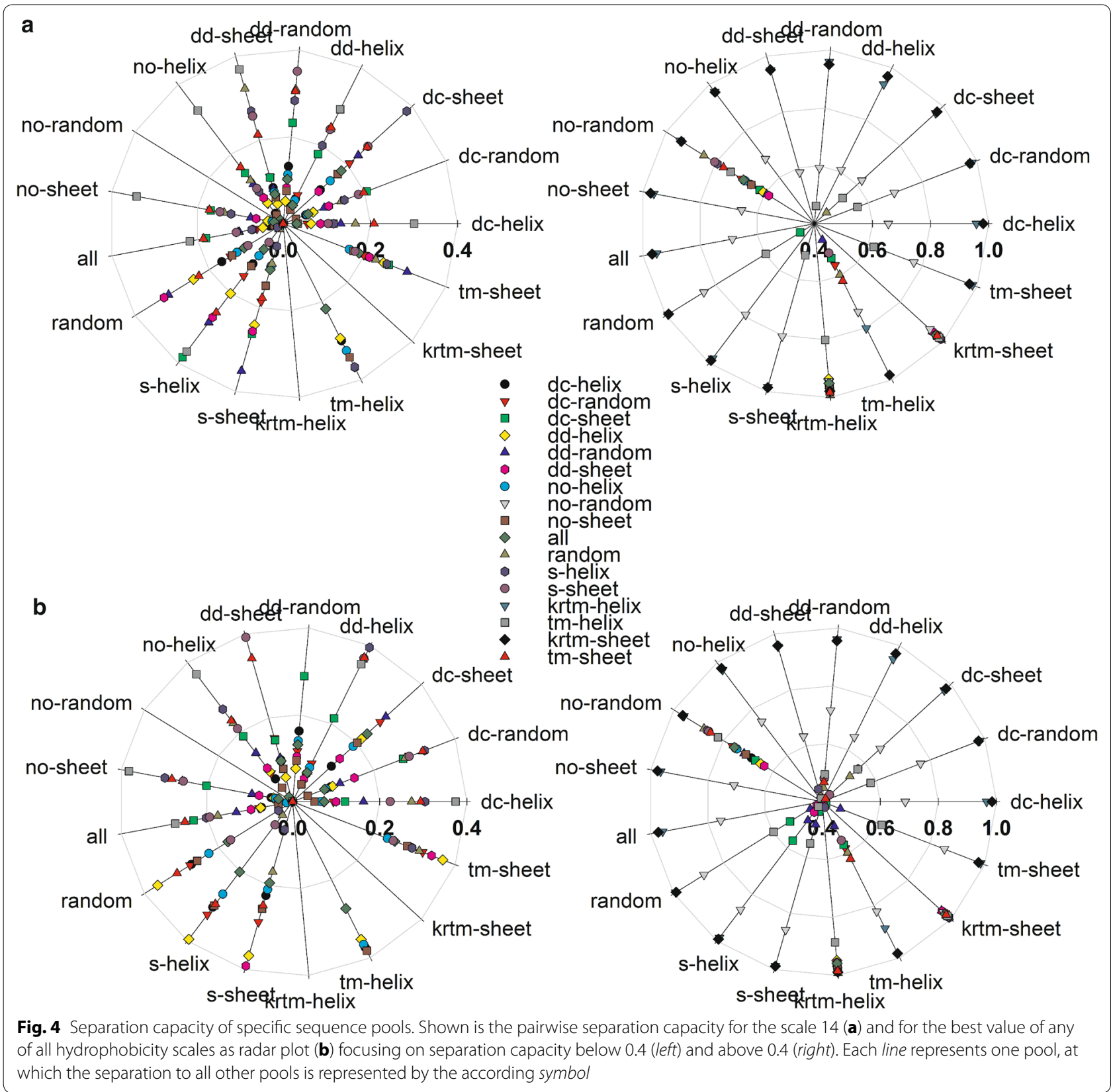

the evolved scale or for scales with hydrophobicity values for Cys, Met, Lys, Val or Ile distinct from the evolved scale (Additional file 8: Fig. S3). Thus, the hydrophobicity value of some amino acids like Gln, His, Gly, Ser or Arg might be more important for the separation capacity of the scales than others.

Thirdly, we asked whether cluster of amino acids with comparable or rather distinct values exist within one scale, which lead to high $S$ values. To this end we analyzed the difference between hydrophobicity values of amino acids of individual scales, namely of the in silico evolved scale, the experimental hydrophobicity scale with highest (best) and the scale lowest (worst) $\mathrm{S}$ value, respectively. Each scale was normalized as such that the highest hydrophobicity value within the scale was set to one and the lowest hydrophobicity value to zero. Subsequently the difference of hydrophobicity values of the amino acids of one scale was calculated (Additional file 9: Table S6). Finally we analyzed whether a pair of amino acids shows a very small $(<0.1$, Fig. 8 a green field) or very large $(>0.9$, Fig. $8 \mathrm{a}$, red field) difference of the hydrophobicity value within each of the three scales. Finally, we 

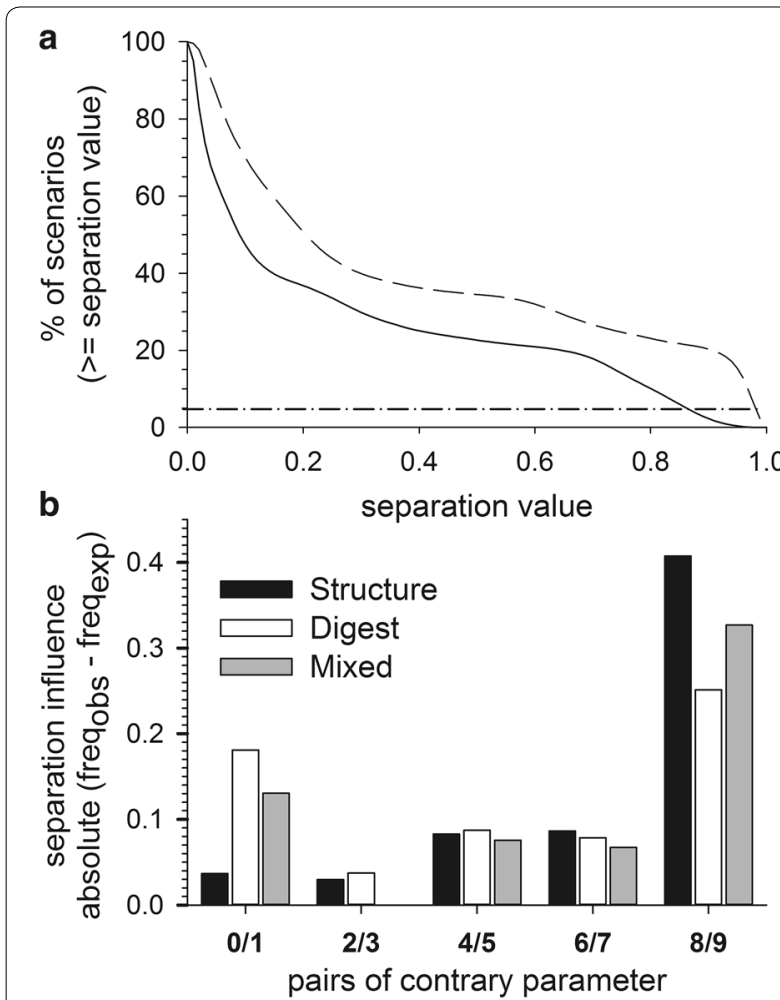

Fig. 5 Influence of hydrophobicity parameter for separation. a Shown is the percentage of scenarios reaching a specific separation values for all sequence pools including outliers (dashed line) and without outliers (solid line). The dash-dotted line shows the best separated $5 \%$ of all scenarios and serves as marginal value to detect the threshold for analyzing the influence of the different hydrophobicity parameter to the separation. $\mathbf{b}$ Shown is the influence on separation of the ten hydrophobicity parameters (Table 5) for the secondary structure based sequence pools (black), the sequence pools generated by digestion (white) and the combination of both (grey). The hydrophobicity parameters are paired (max., min.). The separation influence is calculated as absolute value of the difference between observed and expected frequency of the best $5 \%$ of separated scenarios (Fig. $5 a$ )

inspected which pairs of amino acids show a similar low difference in the experimental scale with highest $S$ value and the evolutionary scale (Fig. 8a, orange frame). In addition, we selected amino acids pairs with very different hydrophobicity value at least in one of the two scales (Fig. 8a, blue frame) and such pairs where the difference was small in one and large in the other one of these two scales (Fig. 8a, yellow frame).

Inspecting the information we realized that a large difference of the hydrophobicity values for glutamate and arginine to each other exists. In addition, the hydrophobicity value of glutamate is most distant to the hydrophobicity values of tyrosine, tryptophan, leucine and isoleucine, respectively Fig. 8a, blue frame). The hydrophobicity value of arginine is distant to the value of

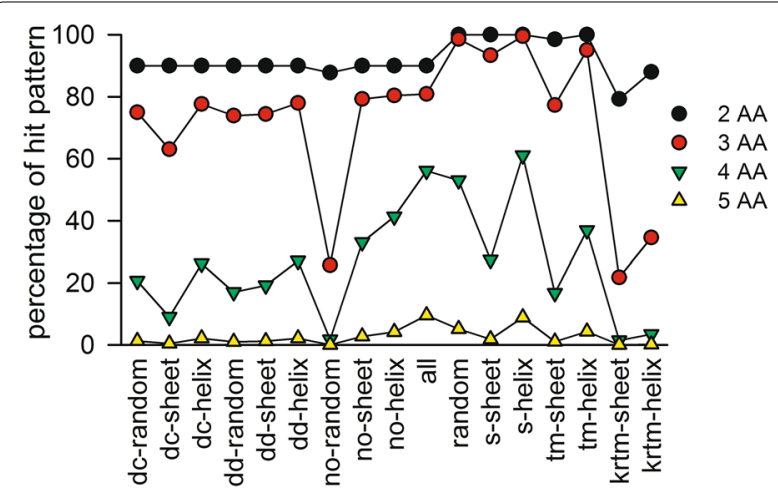

Fig. 6 Amino acid pattern distribution. Shown is the percentage of occurrence of all possible amino acid pattern of a specific length in the different sequence pools. The length of the pattern varies from 2 to 5. 2 AA black circle; 3 AA red circle; 4 AA green triangle down; 5 AA yellow triangle up

phenylalanine and methionine (Fig. 8a, blue frame). In turn, three distinct clusters of amino acids with comparable hydrophobicity values become obvious (Fig. 8a, orange frames). Considering all pairs one can draw relations of the hydrophobicity values within these clusters. Interestingly, the hydrophobicity values of cluster three are most distant form arginine (Fig. 8b), while the hydrophobicity values of cluster one are most distant to glutamate. However, these clusters do not correlate with the amino acid pattern detected for the specific sequence pools (Tables 6, 7) and moreover, they do not necessarily represent the physicochemical properties of the amino acids.

\section{Conclusion}

We demonstrate that most of the hydrophobicity scales reach the same level of peptide separation capacity (Figs. 3,4 ) and thereby, the method by which the scale was generated has no direct influence on clustering or separation capacity (Figs. 1, 3). Nevertheless, if at all we realized that the scale 14 defined by Naderi-Manesh developed in 2001 [40] performs somewhat better than the other hydrophobicity scales. We propose a rule of thumb for experimentalists that aim to use a hydrophobicity scale for identification of peptides with transmembrane segments from a pool of peptides. The hydrophobicity value of arginine and tyrosine should be most distant from the value of glutamate, while the hydrophobicity values of Asn, Asp, His, Lys should be in the center of the scale (Fig. 8c). We further observed that separation of sequence pools defined by known secondary structures is more likely than separation of sequence pools with a combination of secondary structures derived from in silico digestion (Figs. 3, 4), but 
Table 6 Patterns of four amino acids

\begin{tabular}{|c|c|c|c|c|}
\hline Sequence pool & Peptide & FO in pool & $\begin{array}{l}\text { Average } \mathrm{FO} \text { in } \\
\text { remaining set }\end{array}$ & $\begin{array}{l}\text { Average FO in remaining } \\
\text { set GBSS }\end{array}$ \\
\hline \multirow[t]{3}{*}{ dc-helix } & ALAA & 0.0005088 & 0.0001336 & 0.0001327 \\
\hline & AALA & 0.0004970 & 0.0001545 & 0.0001559 \\
\hline & ALLE & 0.0003787 & $6.55 e-05$ & $6.82 e-05$ \\
\hline \multirow[t]{5}{*}{ dc-random } & GSSG & 0.0028090 & 0.0002055 & 0.0001760 \\
\hline & SSGS & 0.0015990 & 0.0001405 & 0.0001205 \\
\hline & SGSS & 0.0014350 & 0.0001022 & $7.90 e-05$ \\
\hline & $\mathrm{HHHH}$ & 0.0007792 & $4.17 e-05$ & $2.41 e-05$ \\
\hline & EEEE & 0.0003691 & $2.28 \mathrm{e}-05$ & $1.88 \mathrm{e}-05$ \\
\hline dc-sheet & VLLV & 0.0003481 & 0.0001082 & 0.0001198 \\
\hline \multirow[t]{2}{*}{ dd-helix } & $E E L L^{*}$ & 0.0003622 & $5.37 e-05$ & $5.67 e-05$ \\
\hline & $\angle E E L^{*}$ & 0.0003372 & $6.47 e-05$ & $6.58 e-05$ \\
\hline \multirow[t]{4}{*}{ dd-random } & GSSG & 0.0006914 & 0.0003379 & 0.0003685 \\
\hline & SSGS & 0.0003872 & 0.0002163 & 0.0002307 \\
\hline & SGSS & 0.0003042 & 0.0001729 & 0.0001818 \\
\hline & SSGL & 0.0002489 & $4.35 e-05$ & $4.62 e-05$ \\
\hline \multirow[t]{3}{*}{ dd-sheet } & GEW ${ }^{*}$ & 0.0002647 & $4.57 e-05$ & $4.93 e-05$ \\
\hline & $P D G T^{*}$ & 0.0002427 & $1.19 e-05$ & $1.53 e-05$ \\
\hline & $D G S V^{*}$ & 0.0002427 & $2.88 e-05$ & $3.05 e-05$ \\
\hline \multirow[t]{2}{*}{ no-helix } & SGSS & 0.0001950 & 0.0001797 & 0.0001918 \\
\hline & PDGS & 0.0001509 & $2.59 e-05$ & $3.24 \mathrm{e}-05$ \\
\hline \multirow[t]{2}{*}{ no-random } & WGI & 0.0017010 & $4.14 \mathrm{e}-05$ & $3.84 \mathrm{e}-05$ \\
\hline & QELD & 0.0010210 & $1.13 e-05$ & $1.45 e-05$ \\
\hline no-sheet & LEAL & 0.0003080 & $8.56 \mathrm{e}-05$ & $9.38 \mathrm{e}-05$ \\
\hline \multirow[t]{5}{*}{ random } & GSSG* & 0.0012510 & 0.0003029 & $2.53 e-05$ \\
\hline & GPSS & 0.0007403 & 0.0001942 & $4.57 e-05$ \\
\hline & SGPS & 0.0006586 & 0.0001508 & $2.70 e-05$ \\
\hline & SSGS & 0.0005360 & $1.43 e-05$ & $6.00 e-07$ \\
\hline & SGSS & 0.0005156 & $1.14 \mathrm{e}-05$ & $3.60 e-06$ \\
\hline s-sheet & VKVI & 0.0001704 & $2.9 e-06$ & $1.16 \mathrm{e}-05$ \\
\hline \multirow[t]{3}{*}{ krtm-helix } & LGLL & 0.0012460 & $6.78 \mathrm{e}-05$ & $5.58 \mathrm{e}-05$ \\
\hline & VLLV & 0.0009341 & $7.16 e-05$ & $6.66 \mathrm{e}-05$ \\
\hline & GIAL & 0.0009341 & $5.29 e-05$ & $4.11 e-05$ \\
\hline \multirow[t]{4}{*}{ tm-helix } & LLLL & 0.0004439 & $7.87 e-05$ & $4.07 e-05$ \\
\hline & LILL & 0.0004040 & $3.21 \mathrm{e}-05$ & $1.25 \mathrm{e}-05$ \\
\hline & LLLV & 0.0003990 & $4.38 \mathrm{e}-05$ & $2.15 \mathrm{e}-05$ \\
\hline & ILLL & 0.0003891 & $2.98 \mathrm{e}-05$ & $1.73 e-05$ \\
\hline krtm-sheet & SIGA & 0.0012020 & $2.92 \mathrm{e}-05$ & $1.69 e-05$ \\
\hline \multirow[t]{3}{*}{ tm-sheet } & SGPL & 0.0004559 & $1.88 \mathrm{e}-05$ & $1.20 \mathrm{e}-05$ \\
\hline & SLNL & 0.0004079 & $1.96 \mathrm{e}-05$ & $2.15 \mathrm{e}-05$ \\
\hline & LYGG & 0.0004079 & $1.06 \mathrm{e}-05$ & $9.80 \mathrm{e}-06$ \\
\hline
\end{tabular}

Given are the sequence pool name (column 1) and peptide sequence with the highest frequency of occurrence (column 2); the frequency of occurrence (FO) of this peptide in the according pool (column 3), the frequency of occurrence of this peptide in the pool containing all sequences except the one of the analyzed pool (column 4), the frequency of occurrence of this peptide in the pool containing all sequences generated by the same strategy (GBSS) as the analyzed pool excluding the sequences of the analyzed pool (column 5). Italic shows peptides that have an at least 50 -fold higher frequency, with respect to the remaining sets (column 4 ) or the remaining peptides generated by the same strategy (column 5). Peptide sequences with p values below 0.05 were marked by an asterisk

the tryptic digested sequence pools with helical and strand content or transmembrane $\beta$-strand or $\alpha$-helix content are best separable from the other pools (Fig. 4).
Nevertheless, we realized a threshold of $\mathrm{S}=0.6$, irrespective of the nature of the scale, which is supported by an in silico approach to optimize the scale (Fig. 7). In turn, the 
Table 7 Amino acid patterns of five amino acids

\begin{tabular}{|c|c|c|c|c|}
\hline Sequence pool & Peptide & FO in pool & FO in remaining set & FO in remaining set GBSS \\
\hline \multirow[t]{4}{*}{ dc-helix } & ALLDA & 0.0001311 & $5.50 e-06$ & $3.50 e-06$ \\
\hline & AALAA & 0.0001311 & $3.75 e-05$ & $4.50 e-05$ \\
\hline & ALDAA & 0.0001180 & $6.30 \mathrm{e}-06$ & $5.50 \mathrm{e}-06$ \\
\hline & AAALA & 0.0001180 & $1.31 \mathrm{e}-05$ & $1.15 e-05$ \\
\hline \multirow[t]{4}{*}{ dc-random } & GSSGS & 0.0016490 & $9.41 e-05$ & $7.02 \mathrm{e}-05$ \\
\hline & SGSSG & 0.0015360 & $8.46 e-05$ & $6.15 e-05$ \\
\hline & SSGSS & 0.0015130 & $8.75 e-05$ & $6.31 e-05$ \\
\hline & $\mathrm{HHHHH}$ & 0.0005872 & $2.50 \mathrm{e}-05$ & $1.33 e-05$ \\
\hline \multirow[t]{4}{*}{ dc-sheet } & VLVNA & 0.0001966 & $4.80 e-06$ & $1.10 e-06$ \\
\hline & SDTV & 0.0001966 & $2.50 e-06$ & $3.20 e-06$ \\
\hline & KGTVT & 0.0001966 & $2.50 e-06$ & $2.20 e-06$ \\
\hline & YLVNM" $^{\#}$ & 0.0001311 & $<1.00 e-08$ & $<1.00 e-08$ \\
\hline \multirow[t]{3}{*}{ dd-helix } & LTEEE & $8.01 \mathrm{e}-05$ & $2.10 e-06$ & $2.10 e-06$ \\
\hline & LTLEE & $9.34 \mathrm{e}-05$ & $5.20 \mathrm{e}-06$ & $7.30 \mathrm{e}-06$ \\
\hline & ELLAD & $8.01 e-05$ & $7.50 \mathrm{e}-06$ & $9.20 \mathrm{e}-06$ \\
\hline \multirow[t]{5}{*}{ dd-random } & GSSGS & 0.0003547 & 0.0001750 & 0.0001879 \\
\hline & SGSSG & 0.0003252 & 0.0001603 & 0.0001716 \\
\hline & SSGSS & 0.0003252 & 0.0001618 & 0.0001711 \\
\hline & GSSGL & 0.0001774 & $1.28 \mathrm{e}-05$ & $1.35 \mathrm{e}-05$ \\
\hline & TILPL \# & 0.0001182 & $1.20 e-06$ & $2.00 e-07$ \\
\hline \multirow[t]{3}{*}{ dd-sheet } & TLDGG $^{\#}$ & 0.0001182 & $4.50 e-06$ & $2.00 e-07$ \\
\hline & SVIDT & $9.52 \mathrm{e}-05$ & $1.40 e-06$ & $2.10 e-06$ \\
\hline & LTVTG $^{\#}$ & $9.52 \mathrm{e}-05$ & $2.80 \mathrm{e}-06$ & $2.00 e-07$ \\
\hline no-helix & GDSGG & $6.76 e-05$ & $2.20 \mathrm{e}-06$ & $3.20 \mathrm{e}-06$ \\
\hline \multirow[t]{2}{*}{ no-random } & VGIVT ${ }^{\#}$ & 0.0011290 & $5.00 e-07$ & $2.00 e-07$ \\
\hline & TGHSL ${ }^{\#}$ & 0.0007524 & $1.20 e-06$ & $1.70 e-06$ \\
\hline \multirow[t]{2}{*}{ no-sheet } & SSGSS & 0.0002080 & 0.0001691 & 0.0001817 \\
\hline & SGSSG & 0.0001976 & 0.0001683 & 0.0001832 \\
\hline \multirow[t]{5}{*}{ all } & VIGGG & $4.35 \mathrm{e}-05$ & $3.90 \mathrm{e}-06$ & $2.70 e-06$ \\
\hline & $\| G G G$ & $3.84 \mathrm{e}-05$ & $2.50 \mathrm{e}-06$ & $2.70 e-06$ \\
\hline & LADAG & $3.07 e-05$ & $2.00 e-06$ & $2.40 \mathrm{e}-06$ \\
\hline & IVGAG & $3.07 e-05$ & $4.80 e-06$ & $6.00 \mathrm{e}-06$ \\
\hline & GVDVV & $3.07 e-05$ & $1.20 \mathrm{e}-06$ & $1.20 \mathrm{e}-06$ \\
\hline \multirow[t]{5}{*}{ random } & SGPSS ${ }^{\#}$ & 0.0005350 & $2.00 e-07$ & $<1.00 e-08$ \\
\hline & GSSGS\# & 0.0007301 & 0.0001515 & $7.00 e-07$ \\
\hline & SGSSG $^{\#}$ & 0.0006744 & 0.0001385 & $7.00 e-07$ \\
\hline & SSGSS & 0.0006744 & 0.0001399 & $8.00 e-06$ \\
\hline & $\mathrm{HHHHH}$ & 0.0002508 & $4.60 e-05$ & $7.00 e-07$ \\
\hline s-helix & EELKK ${ }^{\#}$ & $5.49 e-05$ & $<1.00 e-08$ & $<1.00 e-08$ \\
\hline \multirow[t]{3}{*}{ s-sheet } & CGGSL $^{\#}$ & 0.0001124 & $6.60 e-06$ & $<1.00 e-08$ \\
\hline & GIVSW & $8.03 e-05$ & $4.80 e-06$ & $1.30 e-06$ \\
\hline & YGGVT" & $6.42 \mathrm{e}-05$ & $1.01 \mathrm{e}-05$ & $<1.00 e-08$ \\
\hline \multirow[t]{5}{*}{ krtm-helix } & LLVGI & 0.0004832 & $4.60 e-06$ & $2.60 e-06$ \\
\hline & LAAVA & 0.0004832 & $7.20 e-06$ & $3.10 e-06$ \\
\hline & FLAVL & 0.0004832 & $3.00 e-06$ & $1.20 e-06$ \\
\hline & YVFFG $^{\#}$ & 0.0003221 & $7.00 e-07$ & $<1.00 e-08$ \\
\hline & YPIVW & 0.0003221 & $5.40 e-06$ & $6.00 e-06$ \\
\hline \multirow[t]{2}{*}{ tm-helix } & LILLL & $9.97 e-05$ & $1.00 e-06$ & $7.00 e-07$ \\
\hline & LLLLV & $8.92 e-05$ & $3.10 e-06$ & $4.70 e-06$ \\
\hline
\end{tabular}


Table 7 continued

\begin{tabular}{lllll}
\hline Sequence pool & Peptide & FO in pool & FO in remaining set & FO in remaining set GBSS \\
\hline $\mathrm{krtm}^{-s h e e t}$ & TGTLE & $1.05 e-05$ & $5.40 e-06$ & $6.30 e-06$ \\
$\mathrm{tm}$-sheet & PTLDL & 0.0001878 & $2.77 \mathrm{e}-05$ & $<1.00 e-08$ \\
& LYGKV $^{\#}$ & 0.0001610 & $<1.00 e-08$ & $<1.00 e-08$ \\
& SASAG & $1.40 e-06$ & $<1.00 e-08$ \\
& RQFNV & 0.0001342 & $2.20 e-06$ & $1.40 e-06$
\end{tabular}

Given are the sequence pool name (column 1) and sequence with the highest frequency of occurrence (column 2); the frequency of occurrence (FO) of this peptide in the according pool (column 3), the FO in the pool containing all sequences except the one of the analyzed pool (column 4 ), the FO in the pool containing all sequences generated by the same strategy (GBSS) excluding sequences of the analyzed pool (column 5). Italic shows peptides with at least 50 -fold higher frequency with respect to all (column 4) or peptides of the same strategy (column 5). Hashtag after the pattern indicate 500 -fold higher frequency in at least column 4 or column 5

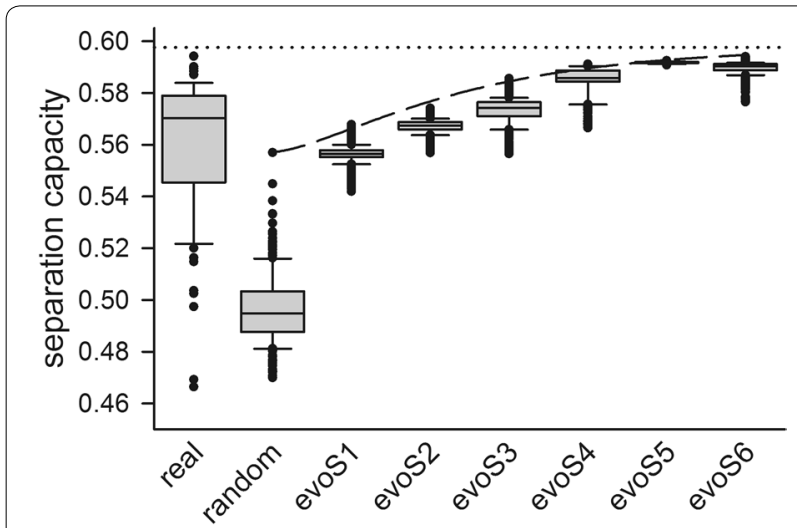

Fig. 7 Separation capacity using evolution of random in silico scales. Shown is the box plot of separation capacity distribution of the 98 real hydrophobicity scales (real), the 200 randomly created scales (random, see "Methods" section) and the six in silico evolution steps (evoS1 to evoS6). The evolutionary optimization of the evolutionary approach was analyzed for the best performing scale identified after each step (dashed line) and the predicted plateau of 0.588 is shown as dotted line

separation capacity depends on the number of parameter calculated (Additional file 3: Fig. S1), although we realized that the alternating hydrophobicity has the lowest capacity for sequence pool separation (Fig. 5). Remarkably, we observed that detection of $B$-strands in peptides can be supported by the detection of penta-peptides (Fig. 6) because such peptides have been detected in the structural pool and in the pools generated by simulated tryptic digest (Table 7). Similarly, amino acid patterns specific for the transmembrane $\beta$-strands (Tables 6, 7) or largely random content (Table 7 ) have been observed. In turn, for pools mainly consisting of helical structures only one specific penta-peptide for soluble (s-helix) and transmembrane (krtm-helix) $\alpha$-helices could be detected (Table 7). Summarizing, the quality of separation of

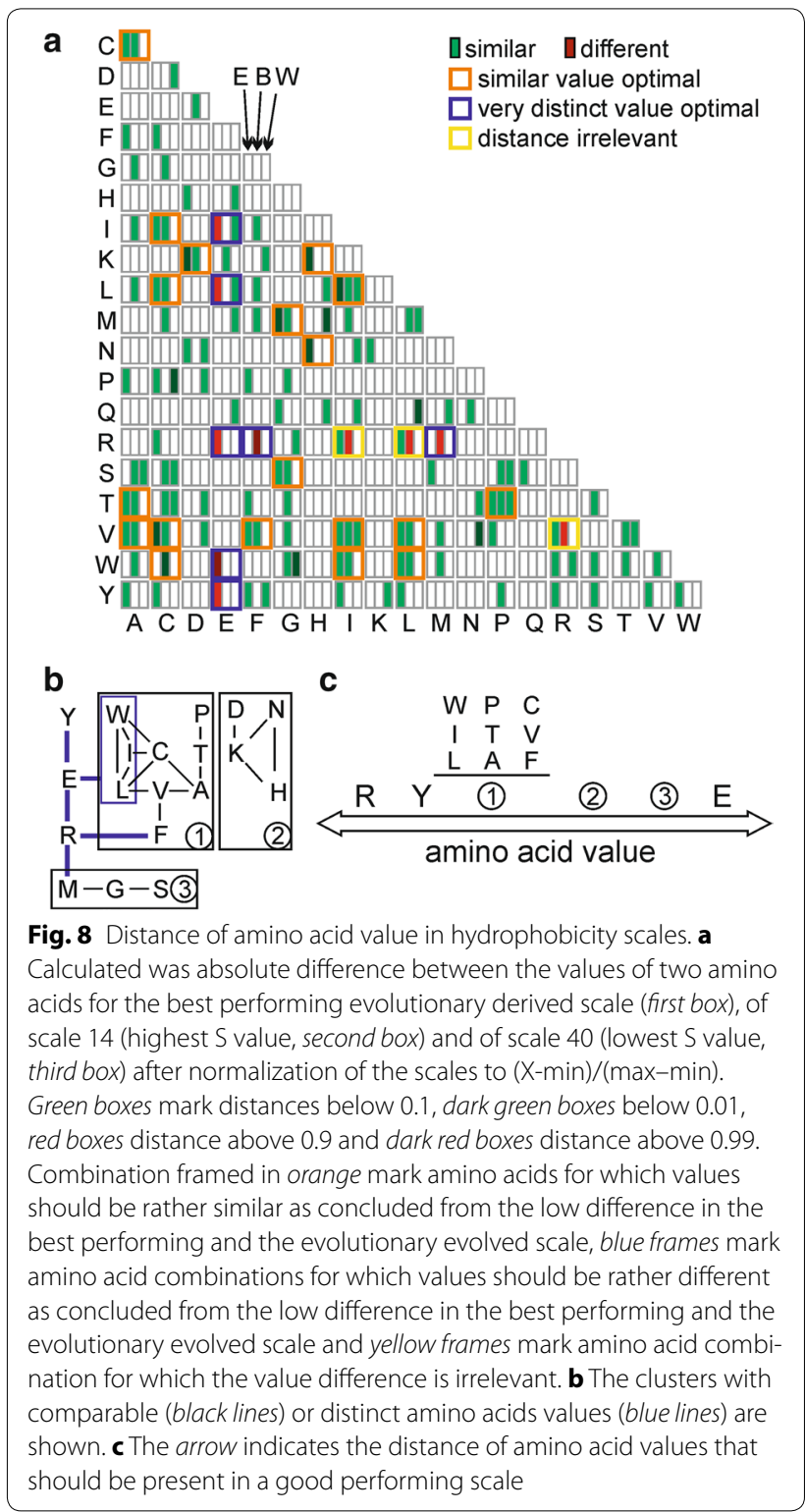


sequence pools depends rather on the parameter used for calculation than on the scale used and can be supported by the search for specific amino acid pattern.

\section{Methods}

\section{Hydrophobicity scales}

98 hydrophobicity scales (Tables 3,4$)-16$ are only reversed algebraic figures of other scales in the set were extracted from three different sources (http://www. genome.jp/aaindex/ [30]; http://split4.pmfst.hr/split/ scales.html [22]; http://web.expasy.org/protscale/ [31]). The path of hydrophobicity scales development is given in Additional file 10: Fig. S4.

\section{Hydrophobicity parameter}

Five different hydrophobicity parameters (Table 5) were used to analyze their influence on the separation capacity. For all of those hydrophobicity parameters we used contrary pairs (max. and min.) of the parameters to look for potential differences. The EBBS [16] should be able to detect $\beta$-sheets, whereas the alternating hydrophobicity $[14,15]$ should be more specific to detect transmembrane $\beta$-sheets. The hydrophobicity moment $\alpha$ and $\beta$ [13] were used to identify $\alpha$-helices and $\beta$-sheet in general. The last parameter was the average hydrophobicity, which had no preferentially detectable secondary structure so far.

\section{Structure pools}

The known secondary structure pools (Tables 1,2$)$ were extracted from the ASTRAL40 database [32] and differed in random coil (random), cytosolic $\beta$-sheets (s-sheet), cytosolic $\alpha$-helix (s-helix), transmembrane $\beta$-sheet (tmsheet) and transmembrane $\alpha$-helix (tm-helix). Further, we implemented an in silico tryptic digest approach to split sequences after Lysine (K) and Arginine (R) of the whole ASTRAL database and classified the peptide fragments concerning their secondary structures. These were divided in fragments containing a (i) continuous dominating SSE (dc), (ii) discontinuous dominating SSE (dd), (iii) no dominating SSE but only two different structures (no-), (iv) all three secondary structures (all) or (v) transmembrane sheet or helix fragments (krtm-).

\section{Pool separation via hydrophobicity scales and parameter Cloud algorithm}

The algorithm to calculate the cloud is a two-step approach. All single peptide sequences of a specific secondary structure dissection pool (Table 1) or in silico K/R-digestion pool (Table 2) were used as input to calculate the cloud. Thereby, each peptide is represented by an $\mathrm{n}$-dimensional vector (hyper volume; convex cover), where the values of the different hydrophobicity parameters ( $\mathrm{n} \leq 5$ dimensions; represented by the minimum or maximum value using a specific hydrophobicity parameter from Table 5) calculated based on a specific hydrophobicity scale (Tables 3,4 ) are the components of this vector.

(I) The initial cloud is calculated based on a randomly chosen as subset of $\sim 30$ points (peptides defined by vectors). Then, the cloud is expanded until each point is considered. In general, the algorithm calculates all distances and directions within the $\mathrm{n}$-dimensional space between all given points (peptides) and tests if these sites are valid. A site is valid if all points of the entire cloud follow the direction of the hypersurface. By this it is determined if an added point lies within the so far calculated cloud.

(II) The existing cloud is expanded point by point to determine the cloud by a set of sites between points. After each point the set of sites is updated by the procedure (i) and all remaining points are tested if these points are inner points or putative boundary points.

(III) The final cloud volume is calculated based on the outer sites between the boundary points in the $\mathrm{n}$ dimensional space that form a convex envelope. All points are placed inside the cloud (inner points) or on the convex envelope (boundary points).

\section{Separation calculation}

All peptides of two structure pools in a given scenario were used to calculate the heuristic hypervolumes of each pool, respectively, defined by the hypersurface via a pipeline. A scenario is defined by the used number of dimensions represented by the selected hydrophobicity parameters, the selection of the hydrophobicity scale and which two structure pools are used for comparison. The number of the points (peptides) positioned by the according vector within the clouds was counted as well as the points within the overlap of both cloud volumes.

\section{Convex envelope}

We remove the boundary points (petides) of both structure pools building the convex envelope to avoid big volumes of the clouds based on outliers. We analyzed the volume and number of peptides per structure pool for all combinations with $\mathrm{n}=5$ dimensions and calculate the loss of peptides and loss of volume in percentage. Due to the high amount of combinations per structure pool (defined by number of hydrophobicity parameter and number of hydrophobicity scales) we calculate the minimum, maximum and average of volume and peptide reduction removing the boundary points (Additional file 11: Table S7). In average, this procedure leads to an elimination of $6.8 \%$ peptide sequences of the structure pool, but a decrease of the according volume of $44.6 \%$. 
By that, removal of putative outlier cause on average a sevenfold increase of the volume per peptide. For pools with low amount of peptides (krtm-sheet, krtm-helix, no-random) the increase of the volume per peptide is lower, namely in the order of twofold. Nevertheless, this increase of density justifies the procedure.

\section{Hydrophobicity scale clustering}

For the hydrophobicity scale clustering the dissimilarity of the different pairs of hydrophobicity values for each amino acid was calculated. This was done by using autocorrelation between all pairs of the 98 different hydrophobicity scales. Afterwards, the Pearson correlation values were normalized to get the dissimilarity and used by MEGA6 [34] to create an UPGMA tree of the dissimilarity. The clustering of the hydrophobicity scales was done by determining a threshold of 0.05 (5\%) for dissimilarity to split the tree in groups.

\section{Amino acid pattern search}

For the amino acid pattern search the different structure pools were used. First, the peptide fragments were analyzed for all occurring amino acid patterns of a specific length based on a Markov chain algorithm of the MEME and MAST suite package (fasta-get-markov) [43]. The algorithm estimates a Markov model from a FASTA file of sequences with previous filtering of ambiguous characters. For example a peptide of four amino acids in length has a conditional probability that one amino acid follows the other amino acid given a specific pool of peptide sequences. So the Markov chain allows the calculation of the transition probability from one state to another state and by this determines the probability of an amino acid occurring in an amino acid peptide of a certain length of a specific pool of peptides. In this approach all possible patterns were detected in the peptides starting from a pattern length of one and incrementing by all different 20 possibilities for each amino acid. The occurrence of the different pattern was normalized to one and compared to the occurrence of the other structure pools to determine the pairwise difference between the pools to detect pool specific pattern of specific length. Furthermore, we performed multiple testing with our identified pattern of length four and five amino acids. We used the Fisher exact test to calculate $p$ values examining the significance of the contingency between occurrences of a specific pattern in relation to a specific structure pool. As reference we pooled all 17 structure pools together. To overcome artificial errors using multiple times the fisher exact test we used as post hoc test Benjamini/Hochberg false discovery rate (fdr) multiple test correction to adjust our $\mathrm{p}$ values (Additional file 5: Table S4, Additional file 6:
Table S5, p values). All amino acid pattern of length four (Table 6) and five (Table 7) with an adjusted p value below $\alpha=0.05$ were marked in bold.

\section{In silico creation of random hydrophobicity scales}

The generation of in silico hydrophobicity scales is based on the minimum and maximum hydrophobicity values extracted out of the 98 analyzed hydrophobicity scales, which were determined as borders for the interval. We used five structure pools to calculate the separation capacity score (dd-sheet, dd-helix, dd-random, krtmsheet, krtm-helix). Two hundred random hydrophobicity scales were created. Based on the best in silico random hydrophobicity scale of the previous steps 2000 scales were created; 100 per amino acid. Half of the hydrophobicity scales per amino acid changed the hydrophobicity value of the single amino acid in the positive [0.001:5] and negative $[-0.001:-5]$ interval (evo1 and evo2). In the following in silico evolution steps (evo3 to evo5) the top 100 newly generated hydrophobicity scales with best performance were analyzed to filter for amino acids which have an influence on the separation capsacity. Only these amino acids were changed in the evo steps evo3 to evo5 to analyze their influence. For evo3 100 hydrophobicity scales per amino acid were created changing within the interval [0.001:10] for $E$ and $Y$ and [-0.001:-10] for A, H, F and L. For evo4 200 hydrophobicity scales per amino acid were created changing within the interval [0.001:20] for E and [-0.001:-20] for A and H. In evo5 400 hydrophobicity scales were created changing within the interval [0.001:40] for E. Finally, in evo6 1000 random hydrophobicity scales based on the best scale of evo5 were created. For each amino acid 25 hydrophobicity scales were created changing within the positive [0.001:5] and 25 scales were created changing within the negative [-0.001:-5] interval.

\section{Additional files}

Additional file 1: Table $\mathbf{S 1 . ~ H y d r o p h o b i c i t y ~ s c a l e ~ v a l u e s . ~ G i v e n ~ i s ~ t h e ~}$ index of the hydrophobicity scale (column 1), the year of publication (column 2), the authors (column 3), the name of the scale (column 4), the website for downloading the scale (column 5) and the hydrophobicity values for each amino acid (columns 6-25).

Additional file 2: Table S2. Correlation of hydrophobicity scales. Given is the correlation matrix of the 98 hydrophobicity scales based on the hydrophobicity values of the 20 amino acids. The name of the scale (column 1 and line 1) as well as the index of the scales (column 2 and line 2) is given.

Additional file 3: Figure S1. Separation capacity using different parameter dimensions. Shown is the separation capacity using a different amount of hydrophobicity parameter ( $3-5$ dimensions, see legend in $B$ ); (A) shows the separation of tryptic digested pools, (B) of secondary structure pools and (C) of all mixed pools. The 98 hydrophobicity scales on the $X$-axis are sorted descending the separation capacity in (B). A separation value of 0 means full-overlap of the pools and 1 means no overlap. 
Additional file 4: Table S3. Amino acid composition in sequence pools. Given are the sequence pool (column 1) and the occurrence of each amino acid in the given pools normalized to 1.

Additional file 5: Table S4. Amino acid pattern of length 4. Given is the sequence pool 1 (column 1) and sequence pool 2 (column 2), the separation capacity between pools based on hydrophobicity (column 3 ), the maximal difference in the frequency of occurrence (FO) pattern value (column 4), the minimal difference in the $\mathrm{FO}$ pattern value (column 5), the number of overrepresented pattern from pool 1 in contrast to pool2 (column 6) and the top 5 of identified pattern of length 4.

Additional file 6: Table S5. Pool specific amino acid pattern of length 5. Given is the sequence pool 1 (column 1) and sequence pool 2 (column 2), the separation capacity between pools based on hydrophobicity (column 3), the maximal difference in the frequency of occurrence (FO) pattern value (column 4), the minimal difference in the FO pattern value (column 5), the number of overrepresented pattern from pool 1 in contrast to pool2 (column 6) and the top 5 of identified pattern of length 5 .

Additional file 7: Figure S2. Normalized amino acid hydrophobicity values of evolved scale. Shown is the normalized hydrophobicity value of all 20 amino acids for the best real (scale 28), worst real (scale 40) corresponding to the five selected sequence pools and in silico evolved hydrophobicity scale as radar plot. Evo scale green squares; best real scale black circles; worst real scale red triangles.

Additional file 8: Figure S3. Correlation of amino acid hydrophobicity distance to evolution scale and separation capacity score of real hydrophobicity scale. Shown is the correlation via linear fit between the separation capacity for the 98 real hydrophobicity scales and the distance of hydrophobicity value of a single amino acid to the in silico evolved scale. The single amino acids are distributed to four graphs (A-D) concerning their slope of the individual linear fit. (A) Raising slope red; (B) slightly raising slope blue; (C) no raising slope black; (D) falling slope green.

Additional file 9: Table S6. Distance of amino acids hydrophobicity values to evolved random scale. Given is the scale identifier (column 1) and scale separation capacity (column 2). Furthermore, for each amino acid (columns 3 to 22) the distance of the normalized hydrophobicity value to the evolved hydrophobicity scale value is shown.

Additional file 10: Figure S4. Organigram of improved hydrophobicity scales. Shown is the relation of hydrophobicity scales with respect to their origin. The dependencies (shown by directed graph) are based on exhaustive literature search. The green marked hydrophobicity scales were included in our study and the red ones not.

Additional file 11: Table S7. Influence of convex envelope on volume and number of peptides. Represented is the reduction of volume and number of peptides per structure pool (column 1; number of all peptides within the pool, column 2) in percentage for all scenarios with $n=5$ dimensions in average (columns 3,4 ), in minimum (columns 5,6 ) and in maximum (columns 7,8 ).

\section{Authors' contributions}

ES and SS conceived and designed the experiments. OM searched the databses for hydrophobicity scales and parameter and used the cloud algorithm. JE created the structure pools via in silico digestion, performed analysis of pool separation and created random hydrophobicity scales. SS performed hydrophobicity scale clustering and amino acid pattern search. ES, SS and JE contribute to write the manuscript. All authors read and approved the final manuscript.

\section{Author details}

${ }^{1}$ Department of Biosciences, Molecular Cell Biology of Plants, Goethe University, Max von Laue Str. 9, 60438 Frankfurt/Main, Germany. ${ }^{2}$ Molecular Bioinformatics, Cluster of Excellence Frankfurt "Macromolecular Complexes", Institute of Computer Science, Faculty of Computer Science and Mathematics,
Goethe-University Frankfurt, Robert-Mayer-Str. 11-15, 60325 Frankfurt/Main, Germany. ${ }^{3}$ Department of Biosciences, Molecular Cell Biology of Plants, Cluster of Excellence Frankfurt (CEF) and Buchmann Institute of Molecular Life Sciences (BMLS), Goethe University, Max von Laue Str. 9, 60438 Frankfurt/Main, Germany.

\section{Acknowledgements}

The work was supported by grants from the Deutsche Forschungsgemeinschaft SFB807-P17 to ES. We thank Nikolaos Konstantinidis, Mario Keller, Katharina Wiesemann and Benjamin Weis for discussion and Johannes UhImann for implementing the tool pipeline for heuristic hyper volume calculation.

\section{Competing interests}

The authors declare that they have no competing interests.

Received: 14 January 2016 Accepted: 17 June 2016

Published online: 04 July 2016

\section{References}

1. Langmuir I. Protein monolayers. Cold Spring Harbor Symp Quant Biol. 1938;1938(6):171-89.

2. Langmuir I. The properties and structure of protein films. Proc Roy Inst Gt Britain. 1938;1938:30483-96.

3. White SH, Wimley WC. Membrane protein folding and stability: physical principles. Annu Rev Biophys Biomol Struct. 1999;1999(28):319-65.

4. Mitaku S, Hirokawa T. Physicochemical factors for discriminating between soluble and membrane proteins: hydrophobicity of helical segments and protein length. Protein Eng. 1999;1999(12):953-7.

5. Sheen SJ. Comparison of chemical and functional properties of soluble leaf proteins from four plant species. J Agric Food Chem. 1991;1991(39):681-5.

6. Tanford C, Lovrien R. Dissociation of Catalase into Subunits. J Am Chem Soc. 1962;1962(84):1892-6.

7. Wilce MCJ, Aguilar MI, Hearn MTW. Physicochemical basis of amino acid hydrophobicity scales: evaluation of four new scales of amino acid hydrophobicity coefficients derived from RP-HPLC of peptides. Anal Chem. 1995;1995(67):1210-9.

8. Ponnuswamy PK, Prabhakaran M, Manavalan P. Hydrophobic packing and spatial arrangement of amino acid residues in globular proteins. Biochim Biophys Acta. 1980;1980(623):301-16.

9. Cid H, Bunster M, Canales M, Gazitúa F. Hydrophobicity and structural classes in proteins. Protein Eng. 1992;1992(5):373-5.

10. Wimley WC, Creamer TP, White SH. Solvation energies of amino acid side chains and backbone in a family of host-guest pentapeptides. Biochemistry. 1996;1996(35):5109-24.

11. Fauchère $J-L$, Pliška $V$. Hydrophobic parameters $\Pi$ of amino-acid side chains from the partitioning of $\mathrm{N}$-acetyl-amino-acid amides. Eur J Med Chem. 1983;18:369-75.

12. Zviling M, Leonov H, Arkin IT. Genetic algorithm-based optimization of hydrophobicity tables. Bioinformatics. 2005;2005(21):2651-6.

13. Eisenberg D, Weiss RM, Terwilliger TC. The hydrophobic moment detects periodicity in protein hydrophobicity. Proc Natl Acad Sci USA. 1984;1984(81):140-4.

14. Mandel-Gutfreund Y, Gregoret LM. On the significance of alternating patterns of polar and non-polar residues in beta-strands. J Mol Biol. 2002;2002(323):453-61.

15. Mirus O, Schleiff E. Prediction of beta-barrel membrane proteins by searching for restricted domains. BMC Bioinformatics. 2005;2005(6):254.

16. Wimley WC. Toward genomic identification of $\beta$-barrel membrane proteins: composition and architecture of known structures. Protein Sci. 2002;2002(11):301-12.

17. Illergård K, Callegari S, Elofsson A. MPRAP: an accessibility predictor for a-helical transmembrane proteins that performs well inside and outside the membrane. BMC Bioinformatics. 2010;2010(11):333.

18. Chen CP, Kernytsky A, Rost B. Transmembrane helix predictions revisited. Protein Sci. 2002;11:2774-91.

19. Pasquier C, Promponas VJ, Palaios GA, Hamodrakas JS, Hamodrakas SJ. A novel method for predicting transmembrane segments in proteins 
based on a statistical analysis of the SwissProt database: the PRED-TMR algorithm. Protein Eng. 1999;1999(12):381-5.

20. Deber CM, Wang C, Liu LP, Prior AS, Agrawal S, Muskat BL, Cuticchia AJ. TM finder: a prediction program for transmembrane protein segments using a combination of hydrophobicity and nonpolar phase helicity scales. Protein Sci. 2001;2001(10):212-9.

21. Juretić $D$, Zoranić $L$, Zucić $D$. Basic charge clusters and predictions of membrane protein topology. J Chem Inf Comput Sci. 2002;2002(42):620-32.

22. Juretić $D$. Protein secondary structure conformations and associated hydrophobicity scales. J Math Chem. 1993;1993(14):35-45.

23. Gromiha MM, Ponnuswamy PK. Prediction of protein secondary structures from their hydrophobic characteristics. Int J Pept Protein Res. 1995;1995(45):225-40.

24. King RD, Sternberg MJE. Identification and application of the concepts important for accurate and reliable protein secondary structure prediction. Protein Sci. 1996;1996(5):2298-310.

25. Cid H, Bunster M, Arriagada E, Campos M. Prediction of secondary structure of proteins by means of hydrophobicity profiles. FEBS Lett. 1982;1982(150):247-54.

26. Peters C, Elofsson A. Why is the biological hydrophobicity scale more accurate than earlier experimental hydrophobicity scales? Proteins. 2014;2014(82):2190-8.

27. Perunov N, England JL. Quantitative theory of hydrophobic effect as a driving force of protein structure. Protein Sci. 2014;2014(23):387-99.

28. Cherry MA, Higgins SK, Melroy H, Lee HS, Pokorny A. Peptides with the same composition, hydrophobicity, and hydrophobic moment bind to phospholipid bilayers with different affinities. J Phys Chem B. 2014;2014(118):12462-70

29. Nicolau DV, Paszek E, Fulga F, Nicolau DV. Mapping hydrophobicity on the protein molecular surface at atom-level resolution. PLoS One. 2014;2014(9):e114042.

30. Kawashima S, Pokarowski P, Pokarowska M, Kolinski A, Katayama T, Kanehisa M. AAindex: amino acid index database, progress report 2008. Nucleic Acids Res. 2008;2008(36):D202-5.

31. Gasteiger E, Hoogland C, Gattiker A, Duvaud S, Wilkins MR, Appel RD, Bairoch $A$. Protein identification and analysis tools on the ExPASy server. In: Walker JM, editor. The proteomics protocols handbook. Totowa: Humana Press Inc.; 2005. p. 571-607.

32. Chandonia JM, Hon G, Walker NS, Lo Conte L, Koehl P, Levitt M, Brenner SE. The ASTRAL compendium in 2004. Nucleic Acids Res. 2004;2004(32):D189-92.

33. Bigelow CC. On the average hydrophobicity of proteins and the relation between it and protein structure. J Theor Biol. 1967:1967(16):187-211.

34. Tamura K, Stecher G, Peterson D, Filipski A, Kumar S. MEGA6: molecular evolutionary genetics analysis version 6.0. Mol Biol Evol. 2013;2013(30):2725-9.

35. Jones DD. Amino acid properties and side-chain orientation in proteins: a cross correlation approach. J Theor Biol. 1975;1975(50):167-83.

36. Zimmerman JM, Eliezer N, Simha R. The characterization of amino acid sequences in proteins by statistical methods. J Theor Biol. 1968;1968(21):170-201.

37. Nozaki Y, Tanford C. The solubility of amino acids and two glycine peptides in aqueous ethanol and dioxane solutions. Establishment of a hydrophobicity scale. J Biol Chem. 1971;1971(246):2211-7.

38. Kyte J, Doolittle RF. A simple method for displaying the hydropathic character of a protein. J Mol Biol. 1982;1982(157):105-32.

39. Engelman DM, Steitz TA, Goldman A. Identifying nonpolar transbilayer helices in amino acid sequences of membrane proteins. Ann Rev of Biophys Biophys Chem. 1986;1986(15):321-53.

40. Naderi-Manesh H, Sadeghi M, Arab S, Moosavi Movahedi AA. Prediction of protein surface accessibility with information theory. Proteins. 2001;2001 (42):452-9.

41. Cornette JL, Cease KB, Margalit H, Spouge JL, Berzofsky JA, DeLisi C. Hydrophobicity scales and computational techniques for detecting amphipathic structures in proteins. J Mol Biol. 1987;1987(195):659-85.

42. Sweet RM, Eisenberg D. Correlation of sequence hydrophobicities measures similarity in three-dimensional protein structure. J Mol Biol. 1983;1983(171):479-88.
43. Bailey TL, Bodén M, Buske FA, Frith M, Grant CE, Clementi L, Ren J, Li WW, Noble WS. MEME SUITE: tools for motif discovery and searching. Nucleic Acids Res. 2009:37:202.

44. Levitt M. Statistical scale of hydrophobicity based on information theory of the observed solvent accessibility of residues in proteins of known structure. J Mol Biol. 1976;1976(104):59-107.

45. Eisenberg D. Three-dimensional structure of membrane and surface proteins. Annu Rev Biochem. 1984;1984(53):595-623.

46. Guy HR. Amino acid side-chain partition energies and distribution of residues in soluble proteins. Biophys J. 1985;1985(47):61-70.

47. Rose GD, Geselowitz AR, Lesser GJ, Lee RH, Zehfus MH. Hydrophobicity of amino acid residues in globular proteins. Science. 1985;1985(229):834-8.

48. Fasman GD. Prediction of protein structure and the principles of protein conformation. New York: Plenum; 1989. p. 789.

49. Chothia C. The nature of the accessible and buried surfaces in proteins. J Mol Biol. 1976;1976(105):1-12.

50. Tanaka S, Scheraga HA. Statistical mechanical treatment of protein conformation. I. Conformational properties of amino acids in proteins. Macromolecules. 1976;1976(9):142-59.

51. Welling GW, Weijer WJ, van der Zee R, Welling-Wester S. Prediction of sequential antigenic regions in proteins. FEBS Lett. 1985;1985(188):215-8.

52. Mohana Rao JK, Argos P. A conformational preference parameter to predict helices in integral membrane proteins. Biochim Biophys Acta. 1986;1986(869):197-214.

53. Bull HB, Breese K. Surface tension of amino acid solutions: a hydrophobicity scale of the amino acid residues. Arch Biochem Biophys. 1974;1974(161):665-70

54. Levitt M. A simplified representation of protein conformations for rapid simulation of protein folding. J Mol Biol. 1976;1976(104):59-107.

55. Bishop CM, Walkenhorst WF, Wimley WC. Folding of beta-sheets in membranes: specificity and promiscuity in peptide model systems. J Mol Biol. 2001;2001(309):975-88.

56. Wimley WC, White SH. Experimentally determined hydrophobicity scale for proteins at membrane interfaces. Nat Struct Biol. 1996;1996(3):842-8.

57. Kuhn LA, Swanson CA, Pique ME, Tainer JA, Getzoff ED. Atomic and residue hydrophilicity in the context of folded protein structures. Proteins. 1995; 1995(23):536-47.

58. Prabhakaran M. The distribution of physical, chemical and conformational properties in signal and nascent peptides. Biochem J. 1990;1990(269):691-6.

59. Roseman MA. Hydrophilicity of polar amino acid side-chains is markedly reduced by flanking peptide bonds. J Mol Biol. 1988;1988(200):513-22.

60. Wolfenden RV, Cullis PM, Southgate CC. Water, protein folding, and the genetic code. Science. 1979;1979(206):575-7.

61. Manavalan P, Ponnuswamy PK. Hydrophobic character of amino acid residues in globular proteins. Nature. 1978;1978(275):673-4.

62. von Heijne G, Blomberg C. Trans-membrane translocation of proteins. The direct transfer model. Eur J Biochem. 1979;1979(97):175-81.

63. Janin J. Surface and inside volumes in globular proteins. Nature. 1979;1979(277):491-2.

64. Wilson KJ, Honegger A, Stötzel RP, Hughes GJ. The behaviour of peptides on reverse-phase supports during high-pressure liquid chromatography. Biochem J. 1981;1981(199):31-41.

65. Argos P, Rao JK, Hargrave PA. Structural prediction of membrane-bound proteins. Eur J Biochem. 1982;1982(128):565-75.

66. Miyazawa S, Jernigan RL. Estimation of effective interresidue contact energies from protein crystal structures: quasi-chemical approximation. Macromolecules. 1985;1985(18):534-52.

67. Jacobs RE, White SH. The nature of the hydrophobic binding of small peptides at the bilayer interface: implications for the insertion of transbilayer helices. Biochemistry. 1989;1989(28):3421-37.

68. Parker JM, Guo D, Hodges RS. New hydrophilicity scale derived from highperformance liquid chromatography peptide retention data: correlation of predicted surface residues with antigenicity and X-ray-derived accessible sites. Biochemistry. 1986;1986(25):5425-32.

69. Cowan R, Whittaker RG. Hydrophobicity indices for amino acid residues as determined by high-performance liquid chromatography. Pept Res. 1990;1990(3):75-80. 
70. Black SD, Mould DR. Development of hydrophobicity parameters to analyze proteins which bear post- or cotranslational modifications. Anal Biochem. 1991;1991(193):72-82.

71. Casari G, Sippl MJ. Structure-derived hydrophobic potential. Hydrophobic potential derived from $X$-ray structures of globular proteins is able to identify native folds. J Mol Biol. 1992;1992(224):725-32.
72. Ponnuswamy PK, Gromiha MM. Prediction of transmembrane helices from hydrophobic characteristics of proteins. Int J Pept Protein Res. 1993;1993(42):326-41.

73. Ponnuswamy PK. Hydrophobic characteristics of folded proteins. Prog Biophys Mol Biol. 1993;1993(59):57-103.

\section{Submit your next manuscript to BioMed Central and we will help you at every step:}

- We accept pre-submission inquiries

- Our selector tool helps you to find the most relevant journal

- We provide round the clock customer support

- Convenient online submission

- Thorough peer review

- Inclusion in PubMed and all major indexing services

- Maximum visibility for your research

Submit your manuscript at

www.biomedcentral.com/submit 